\title{
Validation of pressure drop assessment using 4D flow MRI-based turbulence production in various shapes of aortic stenoses
}

Hojin Ha, John-Peder Escobar Kvitting, Petter Dyverfeldt and Tino Ebbers

The self-archived postprint version of this journal article is available at Linköping University Institutional Repository (DiVA):

http://urn.kb.se/resolve?urn=urn:nbn:se:liu:diva-156013

N.B.: When citing this work, cite the original publication.

Ha, H., Escobar Kvitting, J., Dyverfeldt, P., Ebbers, T., (2019), Validation of pressure drop assessment using 4D flow MRI-based turbulence production in various shapes of aortic stenoses, Magnetic Resonance in Medicine, 81(2), 893-906. https://doi.org/10.1002/mrm.27437

Original publication available at:

https://doi.org/10.1002/mrm.27437

Copyright: Wiley

https://www.wiley.com/en-gb 


\section{Validation of pressure drop assessment using 4D Flow MRI-based turbulence production in various shapes of aortic stenoses}

Hojin $\mathrm{Ha}^{1,2,3}$, John-Peder Escobar Kvitting ${ }^{2,3,4}$, Petter Dyverfeldt ${ }^{2,3}$, Tino Ebbers ${ }^{2,3}$

${ }^{1}$ Department of Mechanical and Biomedical Engineering, Kangwon National University, Chuncheon, Republic of Korea.

2 Division of Cardiovascular Medicine, Department of Medical and Health Sciences, Linköping University, Linköping, Sweden.

${ }^{3}$ Center for Medical Image Science and Visualization (CMIV), Linköping University, Linköping, Sweden.

${ }^{4}$ Department of Cardiothoracic Surgery, Oslo University Hospital, Rikshospitalet, Oslo, Norway.

Running title: 4D Flow MRI-based turbulence production in stenoses.

Number of words: 4954 words

Number of items: 6 Figures, 3 Tables 


\begin{abstract}
Purpose: To validate pressure drop measurements using 4D Flow MRI-based turbulence production in various shapes of stenotic stenoses.

Methods: In-vitro flow phantoms with seven different 3D-printed aortic valve geometries were constructed and scanned with 4D Flow MRI with six-directional flow encoding (ICOSA6). The pressure drop through the valve was non-invasively predicted based on the simplified Bernoulli, the extended Bernoulli, the turbulence production, and the shear-scaling methods. Linear regression and agreement of the predictions with invasively measured pressure drop were analyzed.
\end{abstract}

Results: All pressure drop predictions using 4D Flow MRI were linearly correlated to the true pressure drop but resulted in different regression slopes. The regression slope and 95\% limits of agreement for the simplified Bernoulli method were 1.35 and $11.99 \pm 21.72 \mathrm{mmHg}$. The regression slope and 95\% limits of agreement for the extended Bernoulli method were 1.02 and $0.74 \pm 8.48 \mathrm{mmHg}$. The regression slope and $95 \%$ limits of agreement for the turbulence production method were 0.89 and $0.96 \pm 8.01 \mathrm{mmHg}$. The shear-scaling method presented good correlation with an invasively measured pressure drop, but the regression slope varied between 0.36 and 1.00 depending on the shear-scaling coefficient.

Conclusion: The pressure drop assessment based on the turbulence production method agrees well with the extended Bernoulli method and invasively measured pressure drop in various shapes of the aortic valve. Turbulence-based pressure drop estimation can, as a complement to the conventional Bernoulli method, play a role in the assessment of valve diseases.

Key words: Turbulence; Reynolds stress; pressure drop; magnetic resonance imaging; phase contrast MRI; 4D PC-MRI; 4D Flow MRI 


\section{INTRODUCTION}

Aortic stenosis increases the left ventricular workload as it increases the transvalvular pressure drop per unit cardiac output that the left ventricle generates (1). Assessment of the transvalvular pressure drop, which is referred to as pressure loss or pressure gradient, is recommended by clinical guidelines to determine the severity of a stenosis and to guide treatment strategies (2).

Measuring pressure drop with an intravascular pressure catheter is the gold standard method for pressure drop measurements, however, invasive measurements are not recommended unless non-invasive methods are not available or there appears to be discrepancies between the measured value and the presenting symptoms (2). As a result, noninvasive flow measurements, such as Doppler echocardiography and phase-contrast magnetic resonance imaging (PC-MRI), are the most commonly used methods for estimating pressure drop. Non-invasive pressure drop has been widely estimated based on the simplified Bernoulli equation for both Doppler echocardiography and PC-MRI. The simplified Bernoulli method assumes that the local energy conversion between pressure potential energy and kinetic energy at the constriction is irreversible. The pressure drop is indirectly estimated from a square of the peak blood velocity at the constricted region, which estimates the local energy transfer from pressure to kinetic energy. Doppler echocardiography and PC-MRI have been widely used to measure peak blood velocity at the aortic valve stenosis and to estimate the corresponding pressure drop, due to their non-invasive nature and clinical accessibility (3). Time-resolved, three-directional, and three-dimensional phase-contrast magnetic resonance imaging (which is commonly abbreviated as 4D PC-MRI or 4D Flow MRI) has recently been developed, and clinical applications of 4D Flow MRI for the assessment of pressure drop have been demonstrated based on the method's non-invasive and multi-dimensional velocity assessment $(4,5)$.Despite that the Bernoulli method provides various advantages for pressure drop estimation, discrepancies between Bernoulli-based prediction and invasive pressure measurement are frequently observed due to its inherent underestimation of pressure recovery in the post-stenotic region (6-9). This fact has triggered the necessity for the development of different types of pressure drop estimation that validate and complement the conventional Bernoulli method.

Some new methods for the assessment of pressure drop across a stenosis have recently been proposed based on turbulence quantification of the flow (10-12). Because most of the 
energy loss of the stenotic flow is associated with turbulence in the flow, previous studies have shown that the pressure drop through a constriction can be assessed by quantifying total turbulent kinetic energy (TKE) $(12,13)$. TKE reflects the amount of turbulence in the flow, but as the dissipation is unknown, it is not sufficient to compute the irreversible pressure drop. To estimate total energy dissipation caused by turbulence, Gülan et al. have computed turbulence production by combining TKE measurements with a shear-scaling method (10). In contrast, Ha et al. have directly quantified turbulence production by employing 4D Flow MRI with a six-directional flow encoding sequence (ICOSA6) (11).

Although previous studies have successfully presented the proof-of-concept of turbulence-based pressure drop estimation, they have been limited to few flow conditions with idealized geometries, such as sudden contraction with circular orifice area. Therefore, further validations are needed to demonstrate that the turbulence-based method is valid for various valve shapes, which affect transvalvular peak velocity, effective orifice area, and pressure recovery at the distal region. Demonstrating the robustness of the method and additional experimental evidence are needed before the turbulence-based pressure drop estimation can be introduced in-vivo trials.

In this study, we aimed to validate the estimation of pressure drop using turbulence production methods with various shapes of stenotic heart valves. By employing both direct quantification of turbulence production and the indirect shear-scaling method, we demonstrated the performance of the methods compared with conventional Bernoulli-based and modified Bernoulli-based pressure estimations.

\section{THEORY}

\section{D Flow MRI turbulence quantification theory}

For conventional 4D Flow MRI, the MRI signal $S\left(k_{v}\right)$ for velocity distribution $s(v)$ is expressed by the Fourier-transformation as follows $(14,15)$ :

$$
\mathrm{S}\left(\mathrm{k}_{\mathrm{v}}\right)=\mathrm{C} \int_{-\infty}^{\infty} \mathrm{s}(\mathrm{v}) \mathrm{e}^{-\mathrm{i} \mathrm{k}_{\mathrm{v}} \mathrm{v}} \mathrm{dv}
$$

where $\mathrm{C}$ is a constant scaling factor influenced by the relaxation parameter, spin density, receiver gain, etc. $\mathrm{k}_{\mathrm{v}}$ is the amount of flow sensitivity, which is related to the velocity encoding parameter (VENC) as $\mathrm{k}_{\mathrm{v}}=\pi / \mathrm{VENC}$. When turbulent flow occurs in the region of interest, the intravoxel velocity variance (IVVV) of the turbulent flow along the $i$-direction, $\sigma_{i}^{2}$, can be estimated by the magnitude ratio between the reference signal without velocity 
encoding $\mathrm{S}(0)$ and the signal with velocity encoding along the $i$-direction $\mathrm{S}_{\mathrm{i}}\left(\mathrm{k}_{\mathrm{v}}\right)$ as follows $(14,15)$ :

$$
\sigma_{i}^{2}=\overline{u_{i}^{\prime} u_{i}^{\prime}}=\frac{2}{k_{v}^{2}} \ln \left(\frac{\left|S_{i}(0)\right|}{\left|S_{i}\left(k_{v}\right)\right|}\right),(\mathrm{m} / \mathrm{s})
$$

Here, $u_{i}^{\prime}$ denotes the fluctuating part of the velocity, and - indicates the average operation.

The TKE of the flow can be calculated from IVVV in each direction as follows (16):

$$
T K E=\frac{1}{2} \rho \sum_{i=1}^{3} \sigma_{i}^{2}=\frac{1}{2} \rho\left(\overline{u_{1}^{\prime} u_{1}^{\prime}}+\overline{u_{2}^{\prime} u_{2}^{\prime}}+\overline{u_{3}^{\prime} u_{3}^{\prime}}\right),\left(\mathrm{J} / \mathrm{m}^{3}\right)
$$

where $\rho$ is the density of the fluid.

When velocity encoding direction is distributed in a three-dimensional Cartesian space, the obtained velocity and IVVV can be decomposed into orthogonal components and covariance terms as described in Table 1 . Therefore, three velocity components $(\mathrm{u}, \mathrm{v}$, and $\mathrm{w})$ and the Reynolds stress component tensor $\mathrm{R}_{\mathrm{ij}}$ can be obtained by measuring six nonorthogonal velocity encodings and finding the least square solutions of the six directional phase and magnitude data (17). $\mathrm{R}_{\mathrm{ij}}$ is the six-element symmetric tensor as follow:

$$
R=\left[\begin{array}{lll}
\overline{u_{1}^{\prime} u_{1}^{\prime}} & \overline{u_{1}^{\prime} u_{2}^{\prime}} & \overline{u_{1}^{\prime} u_{3}^{\prime}} \\
\overline{u_{2}^{\prime} u_{1}^{\prime}} & \overline{u_{2}^{\prime} u_{2}^{\prime}} & \overline{u_{2}^{\prime} u_{3}^{\prime}} \\
\overline{u_{3}^{\prime} u_{1}^{\prime}} & \overline{u_{3}^{\prime} u_{2}^{\prime}} & \overline{u_{3}^{\prime} u_{3}^{\prime}}
\end{array}\right]
$$

\section{Direct estimation of turbulence production using ICOSA6}

The actual amount of TKE is a result of the balance between the production of TKE and the dissipation of TKE. Based on the velocity and Reynolds stress obtained from the ICOSA6 sequence, turbulent production $P_{t}$ can be directly calculated as (11):

$$
P_{t}=-R_{i j} S_{i j},(\mathrm{~W} / \mathrm{kg})
$$

where $S_{i j}$ is the strain rate tensor of mean velocity field. The subscripts $i$ and $j$ represent the perpendicular directions $\mathrm{x}, \mathrm{y}$, and $\mathrm{z}$.

\section{Turbulence production estimation using the shear-scaling method}

Based on the recent work by Gülan et al.(10), turbulence production can be also estimated by employing a shear-scaling argument. When the turbulent flow is dominated by the mean shear of the flow, the size of the largest eddies, L, can be expressed as:

$$
L=\frac{c_{\varepsilon}^{S} R_{i i} i^{\frac{1}{2}}}{\left\|s_{i j}\right\|},(\mathrm{m})
$$


where $c_{\varepsilon}^{S}$ is the shear parameter, and $\left\|S_{i j}\right\|$ is the $1_{2}$ norm of the strain rate tensor of the mean velocity field. Then the turbulence production can be equivalent to:

$$
P_{t}=\frac{R_{i i}{ }^{3 / 2}}{L}=c_{\varepsilon}^{s}\left\|S_{i j}\right\| R_{i i},(\mathrm{~W} / \mathrm{kg})
$$

Therefore, the shear-scaling method estimates the turbulence production from the mean strain rate and three diagonal elements of the Reynolds stress tensor, and this method eliminates the need for acquiring off-diagonal Reynolds stress components.

\section{METHODS}

\section{In-vitro flow phantoms}

An in-vitro flow circuit was constructed to simulate valvular flow through a stenotic heart valve (Figure 1a). 3D-printed aortic valves with an inner diameter (D) of $27 \mathrm{~mm}$ were installed in a straight acrylic pipe with an inner diameter of $26 \mathrm{~mm}$. The working fluid (tap water) was circulated through the flow circuit system at steady flow conditions by using a gear pump (ECO Gearchem G6, Pulsafeeder, NY). Four different steady flow rates from 6.8 to $25.2 \mathrm{~L} / \mathrm{min}$ were used to cover the range from mean cardiac output to peak flow rate in the ascending aorta (18). The flow rate was controlled by changing the gear speed of the pump from 300 to 1200 revolutions per minute (RPM), and the exact flow rate was later confirmed from each 4D Flow MRI dataset. The Reynolds numbers of the flow rates were from around 5,552 to 20,000 , which belong to the turbulence flow regime. The use of water as a working fluid was justified since the viscosity effect can be negligible in highly turbulent flows (16). Two pressure ports were installed at $-5 \mathrm{D}$ and $+5 \mathrm{D}$ from the valve level (Figure 1a), and the proximal and distal pressures were directly measured using a digital pressure manometer (GMH-3161-07B, Greisinger, Germany) following previous pressure measurement studies $(6,19)$.

This study tested a total of seven stenotic heart valves: two circular valves mimicking idealized sudden contraction/expansion ( $\mathrm{Circ1}$ and Circ2), one tricuspid aortic valve mimicking three valve commissures that are uniformly closed (TAV), two bicuspid aortic valves mimicking one of the commissures as partially closed (BAV1 and BAV2), and two prosthetic heart valves mimicking one of the leaflets as blocked (PHV1) and both leaflets as partially obstructed (PHV2) (20-22). Stenotic valves were modeled to achieve a geometric orifice area (GOA) of around 0.7 to $2 \mathrm{~cm}^{2}$, mimicking severe to modest aortic valve stenosis 
(Figure $1 \mathrm{~b}$ and also Table 2). Except the idealized circular contraction, TAV, BAV, and PHV valves mimicked possible clinical conditions in patients with varying degrees of aortic stenosis (20-23). Detailed geometries are shown in Figure S1 of the Supporting information.

A total of seven valves were fabricated using an acrylonitrile butadiene styrene-like material (VisiJetM3-X, 3D Systems, Rock Hill, SC) and a 3D printer (Projet 3510 SD, 3D Systems, Rock Hill, SC). Resolution of the system was $32 \mu \mathrm{m}$. Typical accuracy was about $0.025-0.05 \mathrm{~mm}$ per $25.4 \mathrm{~mm}$, which corresponds to $0.1 \%$ to $0.2 \%$ of the valve dimension.

\section{D Flow MRI measurement}

4D flow MRI measurements were performed using a clinical 1.5T MRI scanner (Philips Achieva; Philips Medical Systems, Best, The Netherlands). A conventional gradient-echo sequence with asymmetric four-point flow encoding was modified to have a six-directional icosahedral flow encoding (ICOSA6) and one flow-compensated reference encoding $(11,17,24)$.To maintain the same experimental conditions for all flow rate and valves conditions, the following four different velocity encoding parameter (VENC) were repetitively used: 1, 2, 3, and $5 \mathrm{~m} / \mathrm{s}$. Echo time (TE) and repetition time were 1.5-2.2 $\mathrm{ms}$ and $3.2-3.9 \mathrm{~ms}$, respectively. The flip angle was $10^{\circ}$. The field of view was $336 \mathrm{~mm} \times 336 \mathrm{~mm} \times 57 \mathrm{~mm}$ with a $1.5 \mathrm{~mm}$ isotropic voxel size. Partial echo acquisition with a factor of 0.7 along frequency-encoding directions was used to minimize TE, and the number of signal averages (NSA) was set to five to enhance the signal-to-noise ratio. Scan time for each measurement with an NSA of five was about four minutes (3:36 to 4:25 minutes). Therefore, the total scan time for estimating the turbulence production of each heart valve with four VENC measurements was about 16 minutes.

\section{Post-processing of 4D Flow MRI data}

Estimation of three velocity components and six Reynolds stresses from 4D flow MRI with ICOSA6 encoding was processed as described in previous publications $(11,24)$. Data obtained with VENC $=5 \mathrm{~m} / \mathrm{s}$ were used to extract the velocity of the flow. The velocity field obtained without flow was used to correct the velocity offsets caused by background phase errors (25). After correction for background phase errors, median filtering was applied to smooth the velocity field. Peak velocity, $\mathrm{V}_{\text {peak, }}$, was obtained by finding the maximum velocity in the flow 
region. Flow rate, Q, was obtained at all cross-sections of the flow phantom, and the median value of the obtained flow rates was used in this study.

Since four different VENC parameters from $1 \mathrm{~m} / \mathrm{s}$ to $5 \mathrm{~m} / \mathrm{s}$ were used regardless of flow rate and valve, a multi-VENC method was employed to sum the four datasets and optimize the sensitivity of turbulence (26). At first, the Reynolds stress was reconstructed from the data obtained at $\mathrm{VENC}=1 \mathrm{~m} / \mathrm{s}$. If the flow encoded signal $S_{i}\left(k_{v}\right)$ was lower than three times the noise level at any voxel of the data, where the noise level was obtained by the standard deviation of the magnitude at the inlet flow, the corresponding Reynolds stress data were considered to be underestimated due to MRI noise (14). The Reynolds stress at the corresponding voxel was replaced with the data obtained with higher VENC ( $2 \mathrm{~m} / \mathrm{s})$. If any voxel data obtained at $\mathrm{VENC}=2 \mathrm{~m} / \mathrm{s}$ also showed $S_{i}\left(k_{v}\right)$ lower than three times the noise level, the Reynolds stress at the voxel was again replaced with the data obtained with higher VENC $(3 \mathrm{~m} / \mathrm{s})$. This process was continued until all Reynolds stress data were obtained from the lowest VENC measurement, which provides $S_{i}\left(k_{v}\right)$ higher than three times the noise level.

\section{Bernoulli-based pressure drop estimation}

The following simplified Bernoulli equation has been widely used in medicine:

$$
\Delta \mathrm{P}_{\mathrm{SB}}=4 v_{\text {peak }}^{2},(\mathrm{mmHg})
$$

where $v_{\text {peak }}$ is the velocity at the vena contracta, which was obtained from the maximum velocity in the flow phantom. More recently, the extended Bernoulli equation has been derived for stenotic aortic valves to take into account post-stenotic pressure recovery (27):

$$
\Delta P_{E B}=4 v_{\text {peak }}^{2}\left(1-\frac{E O A}{A_{A}}\right)^{2},(m m H g)
$$

where EOA is the effective orifice area, and $\mathrm{A}_{\mathrm{A}}$ is the area of the aorta. EOA can be obtained from the continuity equation assuming a flat axial velocity profile, $\mathrm{Q}=\mathrm{EOA} \cdot \mathrm{v}_{\text {peak, }}$, where $\mathrm{Q}$ is the flow rate through the flow circuit.

\section{Turbulence-based pressure drop estimation}

Based on the work-energy balance in the flow pipe, the total rate of work and the pressure drop of the flow can be expressed as follow (28):

$$
\mathrm{E}=\mathrm{Q} \Delta \mathrm{P}(\mathrm{W})
$$


where $\mathrm{Q}$ is the flow rate, and $\Delta \mathrm{P}$ is the pressure drop of the flow. Assuming that the total rate of work done by the turbulent flow is mostly dominated by turbulence production, E can be estimated from turbulence production. The product of density and $\mathrm{P}_{\mathrm{t}}$ is the power density of turbulence production with the unit of $\mathrm{W} / \mathrm{m}^{3}$, and its volumetric integration results in the total turbulence production within the region of interest with the unit of $\mathrm{W}$ or $\mathrm{mW}$. Given that fluid kinetic energy $(\mathrm{KE})$ is defined as $\frac{1}{2} \rho v^{2}$, where $\mathrm{v}$ is the fluid velocity, the total work-rate balance in the flow can be described as follows $(11,16,29)$ :

$$
\mathrm{E}=\frac{\partial}{\partial t} \sum_{1}^{N} \frac{1}{2} \rho v^{2} \Delta V+\sum_{1}^{N_{A}} \frac{1}{2} \rho v^{2} v \cdot n \Delta A+\rho \sum_{1}^{N} P_{t} \Delta V, \quad[\mathrm{~W}]
$$

where the first term describes the rate of increase in KE within the control volume, the next describes the convection of $\mathrm{KE}$, and the final term is the total turbulence production. $\mathrm{A}$ and $\mathrm{N}_{\mathrm{A}}$ indicate the surface area of the vessel and the total number of surface elements, respectively. Given that the net contribution of KE is zero for steady pipe flow, the pressure drop due to turbulence production can be expressed as (11):

$$
\Delta \mathrm{P}_{T P}=\frac{\mathrm{E}}{\mathrm{Q}}=\frac{\rho}{Q} \int P_{t} d V,
$$

$\Delta \mathrm{P}_{T P}$ was divided by the coefficient 133.32 to convert the unit of pressure from $\mathrm{Pa}$ to $\mathrm{mmHg}$.

Based on the shear-scaling method from Eq. [5-6], the estimation of pressure drop with shear-scaling was obtained as follow:

$$
\Delta P_{S S}=\frac{\rho}{Q} \int\left(2 v S_{i j} S_{i j}+c_{\varepsilon}^{S}\left\|S_{i j}\right\| R_{i i}\right) d V,
$$

where $v$ is the kinematic viscosity. It is noted that the mean viscous dissipation was added in the first term, and $c_{\varepsilon}^{S}$ was set to 0.1 as the default following a previous study (10). The Bernoulli- and turbulence-based methods are summarized in Figure 2.

\section{Statistical Analysis}

Linear regression was analyzed to assess the relationship between the predicted pressure drop from 4D Flow MRI and the measured pressure drop. The slope and coefficient of determination of the regression line $\left(\mathrm{R}^{2}\right)$ were calculated using RStudio (RStudio, Inc., Boston, MA). Bland-Altman analysis was also used to evaluate the agreement between the prediction and the measurement. 


\section{RESULTS}

\section{Dynamic range of turbulence quantification}

The data quality of the turbulence quantification using 4D Flow MRI was confirmed by analyzing TKE relative to background noise (Figure 3). While a lower VENC measurement provides higher sensitivity for turbulence quantification, it can underestimate peak TKE, mostly because overlying Rician MRI noise underestimates the signal loss due to turbulence. The TKE obtained with $\mathrm{VENC}=1 \mathrm{~m} / \mathrm{s}$ was found to underestimate TKE more pronounced than other VENC measurements, particularly for highly turbulent flows with Circ2, TAV, and BAV2 (Figure 3). In contrast, the lower VENC provides lower noise in TKE in the inlet flow where turbulence is not present (Figure S2 in the Supporting information). The multi-VENC method was found to have the high sensitivity of the low VENC measurement while avoiding TKE underestimation, therefore, the dynamic range of the turbulence quantification was maximized regardless of the valve shape and flow conditions (Figure 3 and Figure $\mathrm{S} 2$ in the Supporting information).The multi-VENC method increased the ratio of maximum TKE to noise $\left(\mathrm{TKE}_{\max } / \mathrm{TKE}_{\text {noise }}\right)$ from $80 \%$ to $246 \%$, depending on the shape of the valve (Figure $4 \mathrm{a}$ ). The high SNR of the TKE measurement was maintained throughout the experiments so that the $\mathrm{TKE}_{\max } / \mathrm{TKE}_{\text {noise }}$ was larger than 32.4 (Figure $4 \mathrm{~b}$ ). Sectional velocity and turbulence production are shown in Figure S3-4 (See also description in the Supporting information).

\section{Comparison of pressure estimations}

Estimations of 4D Flow MRI pressure drops using simplified Bernoulli, extended Bernoulli, turbulence production, and the shear-scaling methods were compared with the experimentally measured pressure drop values. Results showed that all methods linearly correlated to the true pressure drop values (Figure 5 and Table 3). The pressure drops predicted with the simplified Bernoulli method were overestimated compared to the measured pressure values. The slope of the linear regression for the simplified Bernoulli was 1.35 for all data and 1.71 for the data with a pressure drop less than $40 \mathrm{mmHg}\left(\Delta \mathrm{P}_{\text {meas }}<40 \mathrm{mmHg}\right)$. The Bland-Altman mean bias with $95 \%$ limits of agreement was $11.99 \pm 21.72 \mathrm{mmHg}$ for all data and $8.11 \pm$ 13.26mmHgfor $\Delta \mathrm{P}_{\text {meas }}<40 \mathrm{mmHg}$. The pressure predicted with the extended Bernoulli method showed better agreement with the measured pressure values than the simplified Bernoulli method. The slope of the linear regression for the extended Bernoulli method was 1.02 for all data and 1.15 for $\Delta \mathrm{P}_{\text {meas }}<40 \mathrm{mmHg}$. The Bland-Altman mean bias with $95 \%$ limits 
of agreement was $0.74 \pm 8.48 \mathrm{mmHg}$ for all data and $0.45 \pm 6.33 \mathrm{mmHg}$ for $\Delta \mathrm{P}_{\text {meas }}<40 \mathrm{mmHg}$. The pressure drop predicted with the turbulence production method was overestimated at for $\Delta \mathrm{P}_{\text {meas }}<40 \mathrm{mmHg}$, while it was underestimated at $\Delta \mathrm{P}_{\text {meas }} \geq 40 \mathrm{mmHg}$. The slope of the linear regression for the turbulence production method was 0.89 for all data and 0.94 for $\Delta \mathrm{P}_{\text {meas }}<40$ mmHg. The Bland-Altman mean bias with 95\% limits of agreement was $0.96 \pm 8.01 \mathrm{mmHg}$ for all data and $2.25 \pm 5.02 \mathrm{mmHg}$ for $\Delta \mathrm{P}_{\text {meas }}<40 \mathrm{mmHg}$.

The estimated pressure drop with the shear-scaling method with $c_{\varepsilon}^{S}=0.1$ was linearly correlated to the measured pressure values, but it showed a large underestimation. The slope of the linear regression for the shear-scaling method with $c_{\varepsilon}^{S}=0.1$ was 0.36 for all data. The Bland-Altman mean bias with 95\% limits of agreement was $-13.70 \pm 34.66 \mathrm{mmHg}$ (Figure 6). Linear regression analysis between the turbulence production and the shear-scaling parameter in all experimental data provided $c_{\varepsilon}^{S}=0.243$ (Figure $6 \mathrm{~g}$ ). The slope of the linear regression for the shear-scaling method with $c_{\varepsilon}^{S}=0.243$ was 0.88 for all data. The Bland-Altman mean bias with $95 \%$ limits of agreement was $-1.04 \pm 7.82 \mathrm{mmHg}$. Finally, $c_{\varepsilon}^{S}=0.274$ was tested by correcting the regression slope at $c_{\varepsilon}^{S}=0.1$. The slope of the linear regression for the shearscaling method with $c_{\varepsilon}^{S}=0.274$ was 1.00 for all data. The Bland-Altman mean bias with $95 \%$ limits of agreement was $1.70 \pm 5.20 \mathrm{mmHg}$.

\section{DISCUSSION}

This study validated pressure drop assessment using 4D Flow MRI combined with a turbulence production method in various shapes of stenotic heart valves against an experimentally measured true pressure drop. The major findings were that: (1) turbulence production in a broad spectrum of flow rates and stenosis shapes could be quantified with 4D Flow MRI; (2) the pressure drop assessment based on the turbulence production method agreed well with the extended Bernoulli method and invasively measured pressure drop; (3) both Bernoulli-based and turbulence-based methods were linearly correlated to the true pressure drop values, but each method presented a different regression slope and limits of agreement.

This study demonstrated that turbulence-based pressure drop estimation can play a role in validation and can complement the conventional Bernoulli method. While both the extended Bernoulli and turbulence production methods presented good agreements with the 
true pressure drop, they still had limits of agreement greater than $8 \mathrm{mmHg}$. This indicates that clinical decisions can only be safely drawn from either the extended Bernoulli or the turbulence production method if the difference between the predicted pressure drop and the clinical cut-off is greater than $8 \mathrm{mmHg}$. When the difference between the predicted pressure drop and the clinical cut-off is within the limits of agreement, estimations using both the extended Bernoulli and the turbulence-based pressure drop methods may be checked to improve the reliability of the clinical decision.

Although the extended Bernoulli method worked well in the present experiments, we speculate that there are some scenarios in which the turbulence production method can be expected to complement the extended Bernoulli method. First, since the extended Bernoulli method is based on the conventional sudden contraction/expansion flow phenomena (27), it may perform less well in scenarios with a mild-diffusive stenosis, multiple stenoses, and multiple orifices. Carotid artery atherosclerosis, for instance, often develops multiple or diffusive constrictions of the vessel (30). Second, since the extended Bernoulli method assumes inviscid fluid and negligible viscous effects, it may be less accurate for low flow with relatively high viscous effects, such as paradoxical low-flow, low-gradient severe aortic stenosis (31). Since the turbulence production method does not assume the inviscid fluid condition, it may help identify severe stenosis at a low flow condition. Third, the turbulence production method might be beneficial when the stenosis regions cannot be identified. For example, echo-Doppler or phase-contrast MRI measurements of peak velocity measurements of aortic blood flow of mechanical prosthetic heart valves are frequently limited due to metal artifacts. In contrast, turbulence production typically occurs behind the valve region reaching its maximum at the aortic root and ascending aorta (11-13,32). Therefore, the turbulence production method may have advantages for the assessment of stenoses in prosthetic heart valves. However, we acknowledge that none of these scenarios were demonstrated here and remain to be explored in future studies.

While the simplified Bernoulli method is the most widely used clinically (2), it clearly overestimated the true pressure drop compared to the other methods in this study. According to the linear regression, the simplified Bernoulli method overestimates the true pressure drop by about $35 \%$ and even more for pressure drops below $40 \mathrm{mmHg}$. This results in a mean bias and the limits of the agreement $11.99 \pm 21.72 \mathrm{mmHg}$ (Figure 5). Therefore, the true pressure drop and the predicted pressure drop, based on the simplified Bernoulli method are not 
directly interchangeable. The discrepancy between the simplified Bernoulli estimation and the true pressure drop has frequently been reported $(5-8,11)$. Therefore, it functions as a relative metric for clinical cut-off rather than a true pressure prediction (33). Considering the significant amount of clinical data that have been obtained with the simplified Bernoulli method, further methods to make the various pressure estimations interchangeable would be an important issue to investigate in the future.

The shear-scaling method presented a good correlation with the true pressure drop, but the agreements differed with the shear-scaling coefficient. While previous work suggest that $c_{\varepsilon}^{S}=0.1$ can be used for turbulent aortic flow, this resulted in a large underestimation of the true pressure drop in this study (10). According to the regression between the turbulence production method and the shear-scaling method, the shear-scaling parameter of $c_{\varepsilon}^{S}=0.243$ was found to be more accurate and reduce the underestimation of the pressure drop (Figure 6). Considering that previous studies have demonstrated that $c_{\varepsilon}^{S}$ in the turbulent flow is approximately 0.1 regardless of flow rate or valve shape $(10,34,35)$, we suspect that we obtained a different result because the $l_{2}$ norm of the strain rate tensor of the mean velocity field in 4D Flow MRI data was overestimated. 4D Flow MRI velocity fields are affected by noise, and the derived strain rate field depends on the signal-to-noise ratio of the data. Although the velocity and the strain rate have a Gaussian distributed noise with a zero-mean, the square sum and square root of the strain rate provide a non-linear transfer of the Gaussian noise on the strain rate. This leads to an overestimation of the $1_{2}$ norm of the strain rate (24). In addition, estimation of strain rate fields using 4D Flow MRI can also be influenced by the spatial resolution of 4D Flow MRI, which is typically around 2 to $3 \mathrm{~mm}$ (36). Therefore, unless the effects of measurement noise and spatial resolution on the shear-scaling method are clearly identified, the pressure drop prediction of the shear-scaling method cannot be directly interchangeable with other pressure estimations, but would be a relative metric.

This study shows that the noninvasive measurement of Reynolds stress and turbulence production using 4D Flow MRI can be an important tool for analyzing abnormal hemodynamics. Although it is well known that acquired and congenital cardiovascular disorders, such as aortic valve stenosis, contribute to elevated levels of turbulent flow in the aorta (12,37-39), the turbulence in the blood flow and its patho-physiological role are relatively unexplored due to the lack of proper measurement techniques. Various valvular diseases and their effects on hemodynamics have previously been investigated using in-vitro 
experiments or simulations (40-44). Turbulent characteristics of valvular flow have also been investigated using an in-vitro flow phantom with the particle image velocimetry (PIV) technique (45-47). It provided full-field measurement of velocities and Reynolds stresses, and revealed the relationship of turbulent blood flow and pressure loss (41). However, PIV is mostly limited to 2D-plane measurement and requires transparent in-vitro models. While computational fluid dynamics (CFD) is capable of providing highly resolved 3D turbulent blood flow based on patient-specific geometries, the simulation requires experimental verifications and validations to determine if the implementation is correct and if the simulation agrees with physical reality (48-50). In comparison, 4D Flow MRI can provide experimental measurements of blood flow in both in-vitro and in-vivo subjects, therefore, we consider that the noninvasive measurement of turbulence using 4D Flow MRI can play a unique role in revealing the relationship between turbulent blood flow and vascular disease.

We observed an asymmetric jet flow in this study. This can be caused by eccentricity in the 3D-printed valve or eccentricity in the inlet flow profile. A previous simulation study demonstrated that an eccentricity above $0.5 \%$ in the stenosis geometry can generate flow eccentricity in the post-stenosis region (51). However, a distortion of the inlet flow is also known to affect the flow in the post-stenosis region. The curvature or disturbance in the inlet flow can change the inlet velocity profile, which can affect the jet-deflection in the poststenosis region (52). In this study, we used a professional level of 3D printer to manufacture the valves. The typical accuracy of this $3 \mathrm{D}$ printer is about $0.025-0.05 \mathrm{~mm}$ per $25.4 \mathrm{~mm}$, which corresponds to $0.1 \%$ to $0.2 \%$ of the valve dimension. Therefore, the eccentricity of the 3D-printed valve is not expected to be the dominant reason for the jet asymmetry. The jet asymmetry may instead be induced by a non-fully developed inlet velocity profile or an eccentricity in the inlet flow due to experimental imperfection.

Establishing a new measurement technique to use in clinical studies requires several steps of inquiry: Proof-of-concept, in-vitro demonstrations in various experimental conditions, animal studies, and finally clinical studies. We have previously demonstrated the feasibility of turbulence production measurement for estimating irreversible pressure drop estimation as a proof-of-concept study (11). Since the previous study was mostly based on simulation data and included only one ideal valve condition, we aimed to demonstrate in the current study that the method is robust for various valve geometries. Therefore, we validated the turbulence production method for various shapes of stenotic heart valves and compared the performance 
of the method with conventional Bernoulli-based pressure estimations. While the present study may not explain all possible factors regarding the application of the method, it clearly shows that the developed method is valid for various shapes of stenotic heart valves. Therefore, this study is important as it clearly adds experimental evidence in support of the method, which can be a basis for further studies.

One of the limitations of the current study is that the effects of pulsatile flow have not been examined. The use of steady flow reduces the complexity of the study, however, allowing the examination of other desired parameters, such as the effect of the valve shape and flow rate. The use of steady flow also removes the complexity of choosing the proper Bernoulli equation, pressure drop measurement techniques, and temporal resolution related to pulsatile flow measurements. Furthermore, as discussed in (12), flow pulsatility is not expected to affect the performance of the method itself. For pulsatile flow, the same measurements can be done by including the change in kinetic energy (Eq. 10) that takes into account flow acceleration and convection. Therefore, validation in physiological pulsatile flows was not prioritized in the current study. Instead, further studies would investigate on the effects of the pulsatility of the flow, the elasticity of the vessel, and temporal resolution.

It is also noted that the present study used the averaging of the MRI signal and multiVENC analysis to demonstrate the performance of the method at the optimum SNR. When developing a new method, the accuracy of the method at the optimum SNR should be confirmed first. Once the accuracy is established, users can employ various acceleration techniques at the expense of SNR, such as sensitivity encoding (SENSE) and generalized auto calibrating partially parallel acquisitions (GRAPPA) (53-56). Therefore, we did not confine our measurements to scan times that are practical for clinical imaging. In this study, averaging of the MRI signal and multi-VENC analysis to demonstrate the performance of the method at the optimum SNR resulted in a scan time of 16 minutes per each heart valve.

\section{CONCLUSION}

This study validated pressure drop assessment using turbulence production estimation from 4D Flow MRI in various shapes of stenotic heart valves against experimentally measured true pressure drop. The results showed that pressure drop assessment based on the turbulence production method agreed well with the extended Bernoulli method and invasively measured 
pressure drop. The turbulence production method has the potential to function as a complement to the conventional Bernoulli method in the assessment of complex stenoses.

\section{Acknowledgments}

The research leading to these results received funding from the European Union's Seventh Framework Program (FP7/2007-2013) under grant agreement 310612 and the Swedish Research Council; Grant numbers: 2013-6077 and 2014-6191. This study was also supported by a 2018 Research Grant from Kangwon National University (D1001585-01-01).

\section{Competing financial interests}

The author(s) declare no competing financial interests.

\section{REFERENCES}

1. Cioffi G, Faggiano P, Vizzardi E, Tarantini L, Cramariuc D, Gerdts E, de Simone G. Prognostic effect of inappropriately high left ventricular mass in asymptomatic severe aortic stenosis. Heart 2011;97(4):301-307.

2. Baumgartner H, Hung J, Bermejo J, Chambers JB, Evangelista A, Griffin BP, Iung B, Otto CM, Pellikka PA, Quinones M. Echocardiographic Assessment of Valve Stenosis: EAE/ASE Recommendations for Clinical Practice (vol 22, pg 1, 2009). J Am Soc Echocardiog 2009;22(5):442-442.

3. Stamm RB, Martin RP. Quantification of Pressure-Gradients across Stenotic Valves by Doppler Ultrasound. Journal of the American College of Cardiology 1983;2(4):707-718.

4. Donati F, Myerson S, Bissell MM, Smith NP, Neubauer S, Monaghan MJ, Nordsletten DA, Lamata P. Beyond Bernoulli. Circulation: Cardiovascular Imaging 2017;10(1):e005207.

5. Falahatpisheh A, Rickers C, Gabbert D, Heng EL, Stalder A, Kramer HH, Kilner PJ, Kheradvar A. Simplified Bernoulli's method significantly underestimates pulmonary transvalvular pressure drop. Journal of Magnetic Resonance Imaging 2016;43(6):1313-1319.

6. Baumgartner H, Khan S, DeRobertis M, Czer L, Maurer G. Discrepancies between Doppler and catheter gradients in aortic prosthetic valves in vitro. A manifestation of localized gradients and pressure recovery. Circulation 1990;82(4):1467-1475.

7. Levine RA, Jimoh A, Cape EG, McMillan S, Yoganathan AP, Weyman AE. Pressure recovery distal to a stenosis: Potential cause of gradient "verestimation" by Doppler echocardiography. Journal of the American College of Cardiology 1989;13(3):706-715.

8. Niederberger J, Schima H, Maurer G, Baumgartner H. Importance of pressure recovery for the assessment of aortic stenosis by Doppler ultrasound. Circulation 1996;94(8):1934-1940.

9. Vandervoort PM, Greenberg NL, Pu M, Powell KA, Cosgrove DM, Thomas JD. Pressure recovery in bileaflet heart valve prostheses. Circulation 1995;92(12):3464-3472.

10. Gülan U, Binter C, Kozerke S, Holzner M. Shear-scaling-based approach for irreversible energy loss estimation in stenotic aortic flow-An in vitro study. Journal of Biomechanics 2017;56:89-96.

11. Ha H, Lantz J, Ziegler M, Casas B, Karlsson M, Dyverfeldt P, Ebbers T. Estimating the irreversible pressure drop across a stenosis by quantifying turbulence production using $4 \mathrm{D}$ Flow MRI. Scientific Reports 2017;7.

12. Dyverfeldt P, Hope MD, Tseng EE, Saloner D. Magnetic resonance measurement of turbulent 
kinetic energy for the estimation of irreversible pressure loss in aortic stenosis. JACC:

Cardiovascular Imaging 2013;6(1):64-71.

13. Ha H, Kim GB, Kweon J, Huh HK, Lee SJ, Koo HJ, Kang J-W, Lim T-H, Kim D-H, Kim Y-H. Turbulent kinetic energy measurement using phase contrast MRI for estimating the poststenotic pressure drop: in vitro validation and clinical application. PloS one 2016;11(3):e0151540.

14. Dyverfeldt P, Gårdhagen R, Sigfridsson A, Karlsson M, Ebbers T. On MRI turbulence quantification. Magnetic resonance imaging 2009;27(7):913-922.

15. Dyverfeldt P, Sigfridsson A, Kvitting JPE, Ebbers T. Quantification of intravoxel velocity standard deviation and turbulence intensity by generalizing phase-contrast MRI. Magnetic resonance in medicine 2006;56(4):850-858.

16. Pope SB. Turbulent flows: IOP Publishing; 2001.

17. Haraldsson H, Kefayati S, Ahn S, Dyverfeldt P, Lantz J, Karlsson M, Laub G, Ebbers T, Saloner D. Assessment of Reynolds stress components and turbulent pressure loss using 4D flow MRI with extended motion encoding. Magnetic Resonance in Medicine 2017.

18. Powell A, Maier S, Chung T, Geva T. Phase-velocity cine magnetic resonance imaging measurement of pulsatile blood flow in children and young adults: in vitro and in vivo validation. Pediatric cardiology 2000;21(2):104-110.

19. Shandas R, Kwon J, Valdes-Cruz L. A method for determining the reference effective flow areas for mechanical heart valve prostheses. Circulation 2000;101(16):1953-1959.

20. Separham A, Ghaffari S, Aslanabadi N, Sohrabi B, Ghojazadeh M, Anamzadeh E, Hajizadeh R, Davarmoin G. Prosthetic valve thrombosis. Journal of cardiac surgery 2015;30(3):246-250.

21. Pibarot P, Dumesnil JG, Magne J. Prosthetic valve dysfunction. Valvular Heart Disease: Springer; 2009. p 447-473.

22. Han K, Yang DH, Shin SY, Kim N, Kang J-W, Kim D-H, Song J-M, Kang D-H, Song J-K, Kim JB. Subprosthetic pannus after aortic valve replacement surgery: cardiac CT findings and clinical features. Radiology 2015;276(3):724-731.

23. Nishimura RA, Otto CM, Bonow RO, Carabello BA, Erwin JP, Fleisher LA, Jneid H, Mack MJ, McLeod CJ, O'gara PT. 2017 AHA/ACC focused update of the 2014 AHA/ACC guideline for the management of patients with valvular heart disease: a report of the American College of Cardiology/American Heart Association Task Force on Clinical Practice Guidelines. Circulation 2017;135(25):e1159-e1195.

24. Ha H, Lantz J, Haraldsson H, Casas B, Ziegler M, Karlsson M, Saloner D, Dyverfeldt P, Ebbers T. Assessment of turbulent viscous stress using ICOSA 4D Flow MRI for prediction of hemodynamic blood damage. Scientific reports 2016;6.

25. Petersson S, Dyverfeldt P, Sigfridsson A, Lantz J, Carlhäll CJ, Ebbers T. Quantification of turbulence and velocity in stenotic flow using spiral three-dimensional phase-contrast MRI. Magnetic resonance in medicine 2016;75(3):1249-1255.

26. Ha H, Kim GB, Kweon J, Kim YH, Kim N, Yang DH, Lee SJ. Multi-VENC acquisition of four-dimensional phase-contrast MRI to improve precision of velocity field measurement. Magnetic resonance in medicine 2016;75(5):1909-1919.

27. Garcia D, Pibarot P, Dumesnil JG, Sakr F, Durand L-G. Assessment of aortic valve stenosis severity: a new index based on the energy loss concept. Circulation 2000;101(7):765-771.

28. Winter H. Viscous dissipation term in energy equations. Calculation and Measurement Techniques for Momentum, Energy and Mass Transfer 1987;7:27-34.

29. Batchelor GK. An introduction to fluid dynamics: Cambridge university press; 2000.

30. Berger S, Jou L-D. Flows in stenotic vessels. Annual Review of Fluid Mechanics 2000;32(1):347-382.

31. Baumgartner H. Low-flow, low-gradient aortic stenosis with preserved ejection fraction. Journal of the American College of Cardiology 2012;60(14):1268-1270.

32. Dyverfeldt P, Kvitting JPE, Sigfridsson A, Engvall J, Bolger AF, Ebbers T. Assessment of fluctuating velocities in disturbed cardiovascular blood flow: In vivo feasibility of generalized 
phase-contrast MRI. Journal of Magnetic Resonance Imaging 2008;28(3):655-663.

33. Karvandi M. Comments regarding "Simplified Bernoulli's method significantly underestimates pulmonary transvalvular pressure drop". Journal of Magnetic Resonance Imaging 2016;44(3):770-770.

34. Isaza JC, Collins LR. Effect of the shear parameter on velocity-gradient statistics in homogeneous turbulent shear flow. Journal of Fluid Mechanics 2011;678:14-40.

35. Mater BD, Venayagamoorthy SK. A unifying framework for parameterizing stably stratified shear-flow turbulence. Physics of Fluids 2014;26(3):036601.

36. Petersson S, Dyverfeldt P, Ebbers T. Assessment of the accuracy of MRI wall shear stress estimation using numerical simulations. Journal of Magnetic Resonance Imaging 2012;36(1):128-138.

37. Lantz J, Ebbers T, Engvall J, Karlsson M. Numerical and experimental assessment of turbulent kinetic energy in an aortic coarctation. Journal of biomechanics 2013;46(11):1851-1858.

38. Stein PD, Sabbah HN. Turbulent blood flow in the ascending aorta of humans with normal and diseased aortic valves. Circulation research 1976;39(1):58-65.

39. Yamaguchi T, Kikkawa S, Tanishita K, Sugawara M. Spectrum analysis of turbulence in the canine ascending aorta measured with a hot-film anemometer. Journal of biomechanics 1988;21(6):489-495.

40. Lim W, Chew Y, Chew T, Low H. Pulsatile flow studies of a porcine bioprosthetic aortic valve in vitro: PIV measurements and shear-induced blood damage. Journal of biomechanics 2001;34(11):1417-1427.

41. Lim W, Chew Y, Chew T, Low H. Steady flow dynamics of prosthetic aortic heart valves: a comparative evaluation with PIV techniques. Journal of biomechanics 1998;31(5):411-421.

42. Saikrishnan N, Yap C-H, Milligan NC, Vasilyev NV, Yoganathan AP. In vitro characterization of bicuspid aortic valve hemodynamics using particle image velocimetry. Annals of biomedical engineering 2012;40(8):1760-1775.

43. Ge L, Leo H-L, Sotiropoulos F, Yoganathan AP. Flow in a mechanical bileaflet heart valve at laminar and near-peak systole flow rates: CFD simulations and experiments. Journal of biomechanical engineering 2005;127(5):782-797.

44. Yoganathan AP, Chandran K, Sotiropoulos F. Flow in prosthetic heart valves: state-of-the-art and future directions. Annals of biomedical engineering 2005;33(12):1689-1694.

45. Dasi L, Ge L, Simon H, Sotiropoulos F, Yoganathan A. Vorticity dynamics of a bileaflet mechanical heart valve in an axisymmetric aorta. Physics of Fluids (1994-present) 2007;19(6):067105.

46. Miron P, Vétel J, Garon A. On the use of the finite-time Lyapunov exponent to reveal complex flow physics in the wake of a mechanical valve. Experiments in Fluids 2014;55(9):1-15.

47. Yagi T, Yang W, Umezu M. Effect of bileaflet valve orientation on the 3D flow dynamics in the sinus of Valsalva. Journal of Biomechanical Science and Engineering 2011;6(2):64-78.

48. Casey M, Wintergerste T. ERCOFTAC Special Interest Group on "Quality and Trust in Industrial CFD”: Best Practice Guidelines. European Research Community on Flow, Turbulence and Combustion 2000:123.

49. Boutsianis E, Guala M, Olgac U, Wildermuth S, Hoyer K, Ventikos Y, Poulikakos D. CFD and PTV steady flow investigation in an anatomically accurate abdominal aortic aneurysm. Journal of Biomechanical Engineering 2009;131(1):011008.

50. Leuprecht A, Kozerke S, Boesiger P, Perktold K. Blood flow in the human ascending aorta: a combined MRI and CFD study. Journal of engineering mathematics 2003;47(3):387-404.

51. Griffith MD, Leweke T, Thompson MC, Hourigan K. Effect of small asymmetries on axisymmetric stenotic flow. Journal of Fluid Mechanics 2013;721.

52. Peterson SD, Plesniak MW. The influence of inlet velocity profile and secondary flow on pulsatile flow in a model artery with stenosis. Journal of Fluid Mechanics 2008;616:263-301.

53. Pruessmann K, Weiger M, Scheidegger M, Boesiger P. SENSE: sensitivity encoding for fast MRI Magn Reson Med 1999; 42 (5): 952-62. 
54. Lustig M, Donoho D, Pauly JM. Sparse MRI: The application of compressed sensing for rapid MR imaging. Magnetic resonance in medicine 2007;58(6):1182-1195.

55. Otazo R, Kim D, Axel L, Sodickson DK. Combination of compressed sensing and parallel imaging for highly accelerated first-pass cardiac perfusion MRI. Magnetic Resonance in Medicine 2010;64(3):767-776.

56. Griswold MA, Jakob PM, Heidemann RM, Nittka M, Jellus V, Wang J, Kiefer B, Haase A. Generalized autocalibrating partially parallel acquisitions (GRAPPA). Magnetic resonance in medicine 2002;47(6):1202-1210. 


\section{Figure Legends}

Figure 1. Schematics of in-vitro experiments. (a) fluid-circuit system and (b) 3D-printed heart valves for simulating aortic valve stenosis. White dotted lines in (b) indicate fused commissure between valve leaflets. TAV, tricuspid aortic valve; BAV, bicuspid aortic valve; Circ, circular aortic valve; PHV, prosthetic heart valve.

Figure 2. Schematic descriptions of 4D Flow MRI-based pressure estimations. SB, simplified Bernoulli; EB, extended Bernoulli; TP, turbulence production; SS, shear-scaling.

Figure 3. Turbulent kinetic energy (TKE) at various VENC and multi-VENC measurements. TKE at post-valve region $(\mathrm{X} / \mathrm{D}=1)$ was shown.

Figure 4. Dynamic ranges of turbulence measurement (a) dynamic range of turbulence measurement, $\mathrm{TKE}_{\max } / \mathrm{TKE}_{\text {noise, }}$ at various VENC and multi-VENC measurements, (b) dynamic range of turbulence measurement, $\mathrm{TKE}_{\max } / \mathrm{TKE}_{\text {noise, }}$ at four different flow rates. A flow rate of $\sim 25.2 \mathrm{~L} / \mathrm{min}$ was used in (a). All results in (b) were estimated after applying the multi-VENC method.

Figure 5. Comparison of 4D Flow MRI pressure drop estimations. Linear regression between the measured pressure drop and predicted 4D Flow MRI using (a) simplified Bernoulli, (b) extended Bernoulli, and (c) turbulence production methods. Enlarged views of linear regressions between the measured pressure drop and predicted 4D Flow MRI pressure drops using (d) simplified Bernoulli, (e) extended Bernoulli, and (f) turbulence production methods. Bland-Altman analysis between the measured pressure drop and 4D Flow MRI predictions using (g) simplified Bernoulli, (h) extended Bernoulli, and (i) turbulence production methods. SB, simplified Bernoulli; EB, extended Bernoulli; $\mathrm{TP}$, turbulence production.

Figure 6. Demonstration of 4D Flow MRI pressure drop estimation using shear-scaling methods. Linear regression between the measured pressure drop and predicted 4D Flow MRI pressure drops using the shear-scaling method at (a) $C_{\varepsilon}^{S}=0.1$, (b) $C_{\varepsilon}^{S}=0.243$ and (c) $C_{\varepsilon}^{S}=0.274$. Bland-Altman analysis between the measured pressure drop and predicted 4D Flow MRI pressure drops using the shear scaling method at (d) $C_{\varepsilon}^{S}=0.1$, (e) $C_{\varepsilon}^{S}=0.243$, and (f) $C_{\varepsilon}^{S}=0.274$. (g) Comparison between measured turbulence production and prediction with shear-scaling method. SS, shear-scaling. 
Table 1. Summary of in-vitro validation of 4D Flow MRI for pressure drop assessment

\begin{tabular}{clll}
\hline $\begin{array}{c}\text { Number of } \\
\text { encoding }\end{array}$ & Moment direction & Velocity component & Intravoxel variance \\
\hline 1 & $\Delta \mathrm{M}_{1}\left(\cos \theta^{*} \cdot \mathrm{x}+\sin \theta \cdot \mathrm{y}\right)$ & $\mathrm{V}_{1}=\cos \theta \cdot \mathrm{u}+\sin \theta \cdot \mathrm{v}$ & $\sigma_{1}^{2}=\cos ^{2} \theta \cdot\left(\mathrm{R}_{\mathrm{xx}}^{2}\right)+\sin ^{2} \theta \cdot\left(\mathrm{R}_{\mathrm{yy}}{ }^{2}\right)+2 \cos \theta \cdot \sin \theta \cdot \mathrm{R}_{\mathrm{xy}}$ \\
2 & $\Delta \mathrm{M}_{1}(\cos \theta \cdot \mathrm{x}-\sin \theta \cdot \mathrm{y})$ & $\mathrm{V}_{2}=\cos \theta \cdot \mathrm{u}-\sin \theta \cdot \mathrm{v}$ & $\sigma_{2}{ }^{2}=\cos ^{2} \theta \cdot\left(\mathrm{R}_{\mathrm{xx}}{ }^{2}\right)+\sin ^{2} \theta \cdot\left(\mathrm{R}_{\mathrm{yy}}{ }^{2}\right)-2 \cos \theta \cdot \sin \theta \cdot \mathrm{R}_{\mathrm{xy}}$ \\
3 & $\Delta \mathrm{M}_{1}(\cos \theta \cdot \mathrm{y}+\sin \theta \cdot \mathrm{z})$ & $\mathrm{V}_{3}=\cos \theta \cdot \mathrm{v}+\sin \theta \cdot \mathrm{w}$ & $\sigma_{3}{ }^{2}=\cos ^{2} \theta \cdot\left(\mathrm{R}_{\mathrm{yy}}{ }^{2}\right)+\sin ^{2} \theta \cdot\left(\mathrm{R}_{\mathrm{zz}}{ }^{2}\right)+2 \cos \theta \cdot \sin \theta \cdot \mathrm{R}_{\mathrm{yz}}$ \\
4 & $\Delta \mathrm{M}_{1}(\cos \theta \cdot \mathrm{y}-\sin \theta \cdot \mathrm{z})$ & $\mathrm{V}_{4}=\cos \theta \cdot \mathrm{v}-\sin \theta \cdot \mathrm{w}$ & $\sigma_{4}{ }^{2}=\cos ^{2} \theta \cdot\left(\mathrm{R}_{\mathrm{yy}}{ }^{2}\right)+\sin ^{2} \theta \cdot\left(\mathrm{R}_{\mathrm{zz}}{ }^{2}\right)-2 \cdot \cos \theta \cdot \sin \theta \cdot \mathrm{R}_{\mathrm{yz}}$ \\
5 & $\Delta \mathrm{M}_{1}(\sin \theta \cdot \mathrm{x}+\cos \theta \cdot \mathrm{z})$ & $\mathrm{V}_{5}=\sin \theta \cdot \mathrm{u}+\cos \theta \cdot \mathrm{w}$ & $\sigma_{5}{ }^{2}=\sin ^{2} \theta \cdot\left(\mathrm{R}_{\mathrm{xx}}{ }^{2}\right)+\cos ^{2} \theta \cdot\left(\mathrm{R}_{\mathrm{zz}}{ }^{2}\right)+2 \cdot \cos \theta \cdot \sin \theta \cdot \mathrm{R}_{\mathrm{xz}}$ \\
6 & $\Delta \mathrm{M}_{1}(\sin \theta \cdot \mathrm{x}-\cos \theta \cdot \mathrm{z})$ & $\mathrm{V}_{6}=\sin \theta \cdot \mathrm{u}-\cos \theta \cdot \mathrm{w}$ & $\sigma_{6}{ }^{2}=\sin ^{2} \theta \cdot\left(\mathrm{R}_{\mathrm{xx}}{ }^{2}\right)-\cos ^{2} \theta \cdot\left(\mathrm{R}_{\mathrm{zz}}{ }^{2}\right)+2 \cdot \cos \theta \cdot \sin \theta \cdot \mathrm{R}_{\mathrm{xz}}$
\end{tabular}

${ }^{*} \theta$ for the present study is about $31.17^{\circ}$, which corresponds to $\cos \theta=0.8507$ and $\sin \theta=0.5257 . \mathrm{u}, \mathrm{v}$ and $\mathrm{w}$ are velocity components along $\mathrm{x}, \mathrm{y}$, and $\mathrm{z}$, respectively. $\mathrm{R}$ is the Reynolds stress tensor. 
Table 2. Summary of in-vitro validation of 4D Flow MRI for pressure drop assessment

\begin{tabular}{|c|c|c|c|c|c|c|c|c|c|c|c|c|c|c|}
\hline $\begin{array}{c}\text { Valve } \\
\text { Type }\end{array}$ & $\begin{array}{r}\mathbf{Q} \\
{[\mathbf{L} / \mathbf{m i n}]}\end{array}$ & $\begin{array}{r}\text { Peak } \\
\text { Velocity } \\
{[\mathrm{m} / \mathrm{s}]}\end{array}$ & $\begin{array}{l}\text { GOA } \\
{\left[\mathrm{cm}^{2}\right]}\end{array}$ & $\begin{array}{l}\text { EOA } \\
{\left[\mathrm{cm}^{2}\right]}\end{array}$ & $\begin{array}{r}\mathbf{A}_{\mathbf{A}} \\
{\left[\mathbf{m}^{2}\right]}\end{array}$ & $\begin{array}{c}\text { Turbulence } \\
\text { Production } \\
{[\mathrm{mW}]}\end{array}$ & $\begin{array}{l}\text { TKE } \\
{[\mathrm{mJ}]}\end{array}$ & $\begin{array}{r}\Delta \mathbf{P}_{\text {meas }} \\
{[\mathbf{m m H g}]}\end{array}$ & $\begin{array}{r}\Delta \mathbf{P}_{\text {SB }} \\
{[\mathbf{m m H g}]}\end{array}$ & $\begin{array}{r}\Delta \mathbf{P}_{E B} \\
{[\mathbf{m m H g}]}\end{array}$ & $\begin{array}{r}\Delta \mathbf{P}_{\mathrm{TP}} \\
{[\mathbf{m m H g}]}\end{array}$ & $\begin{array}{r}\Delta \mathbf{P}_{\text {ss, }} \mathbf{C}=0.1 \\
{[\mathbf{m m H g}]}\end{array}$ & $\begin{array}{r}\Delta \mathbf{P}_{\mathrm{SS}}, \\
\mathrm{C}=\mathbf{0 . 2 4 3} \\
{[\mathrm{mmHg}]}\end{array}$ & $\begin{array}{r}\Delta \mathbf{P S S}_{\mathrm{sS}} \\
\mathrm{C}=0.274 \\
{[\mathrm{mmHg}]}\end{array}$ \\
\hline Circ1 & 6.8 & 0.9 & 1.8 & 1.3 & & 78.4 & 1.4 & 2.5 & 3.3 & 1.9 & 5.2 & 0.8 & 2.0 & 2.2 \\
\hline Circ1 & 13.8 & 1.7 & 1.8 & 1.4 & & 250.8 & 5.5 & 9.6 & 11.7 & 6.5 & 8.2 & 2.8 & 6.6 & 7.4 \\
\hline Circ1 & 20.8 & 2.6 & 1.8 & 1.4 & & 630.3 & 11.2 & 15.5 & 26.4 & 14.7 & 13.6 & 5.4 & 12.9 & 14.6 \\
\hline Circ1 & 27.2 & 3.3 & 1.8 & 1.4 & & 1297.7 & 19.6 & 21.2 & 43.2 & 23.7 & 21.4 & 8.7 & 20.9 & 23.6 \\
\hline Circ2 & 7.1 & 1.8 & 0.9 & 0.7 & & 192.7 & 3.8 & 5.9 & 12.9 & 9.9 & 12.2 & 3.8 & 9.0 & 10.2 \\
\hline Circ2 & 14.1 & 3.4 & 0.9 & 0.7 & & 929.6 & 13.5 & 25.5 & 45.5 & 34.3 & 29.7 & 12.0 & 29.0 & 32.7 \\
\hline Circ2 & 21.2 & 5.0 & 0.9 & 0.7 & & 2721.0 & 30.8 & 60.1 & 99.3 & 74.5 & 57.8 & 23.7 & 57.3 & 64.6 \\
\hline Circ2 & 25.5 & 5.8 & 0.9 & 0.7 & & 4887.0 & 52.3 & 98.8 & 136.4 & 101.5 & 86.2 & 35.6 & 86.1 & 97.1 \\
\hline TAV & 7.0 & 1.7 & 0.7 & 0.7 & & 188.9 & 4.0 & 4.7 & 11.3 & 8.5 & 12.2 & 3.8 & 9.0 & 10.2 \\
\hline TAV & 13.9 & 3.2 & 0.7 & 0.7 & & 861.8 & 13.2 & 26.0 & 41.0 & 30.6 & 27.9 & 11.0 & 26.6 & 30.0 \\
\hline TAV & 20.6 & 4.7 & 0.7 & 0.7 & & 2898.2 & 34.2 & 63.2 & 89.5 & 66.7 & 63.3 & 25.1 & 60.6 & 68.3 \\
\hline TAV & 25.4 & 5.8 & 0.7 & 0.7 & & 5531.6 & 58.2 & 102.5 & 133.3 & 99.0 & 97.9 & 37.4 & 90.5 & 102.0 \\
\hline BAV1 & 6.8 & 0.9 & 1.6 & 1.3 & & 80.4 & 1.5 & 1.5 & 3.2 & 1.8 & 5.3 & 1.0 & 2.4 & 2.7 \\
\hline BAV1 & 14.1 & 1.7 & 1.6 & 1.4 & 521 & 242.0 & 5.0 & 5.8 & 11.7 & 6.4 & 7.7 & 2.7 & 6.4 & 7.2 \\
\hline BAV1 & 20.9 & 2.4 & 1.6 & 1.4 & 5.31 & 637.5 & 11.0 & 12.6 & 23.8 & 12.7 & 13.7 & 5.4 & 13.0 & 14.6 \\
\hline BAV1 & 27.0 & 3.1 & 1.6 & 1.4 & & 1282.4 & 19.3 & 21.0 & 39.0 & 20.7 & 21.4 & 8.9 & 21.4 & 24.1 \\
\hline BAV2 & 6.7 & 1.3 & 1.0 & 0.8 & & 131.5 & 2.8 & 3.5 & 7.1 & 5.0 & 8.8 & 2.2 & 5.3 & 5.9 \\
\hline BAV2 & 13.4 & 2.4 & 1.0 & 1.0 & & 544.8 & 9.3 & 14.2 & 22.4 & 15.2 & 18.3 & 6.7 & 16.2 & 18.2 \\
\hline BAV2 & 20.2 & 3.6 & 1.0 & 1.0 & & 1568.4 & 20.2 & 29.7 & 50.9 & 34.3 & 34.9 & 13.5 & 32.5 & 36.6 \\
\hline BAV2 & 23.4 & 4.1 & 1.0 & 0.9 & & 2491.4 & 31.0 & 53.5 & 68.9 & 46.6 & 47.9 & 19.0 & 45.8 & 51.7 \\
\hline PVH1 & 6.8 & 0.7 & 2.1 & 1.6 & & 75.1 & 0.7 & 0.8 & 1.9 & 0.9 & 4.9 & 0.5 & 1.1 & 1.2 \\
\hline PVH1 & 13.3 & 1.3 & 2.1 & 1.7 & & 159.8 & 2.8 & 4.2 & 6.7 & 3.1 & 5.4 & 1.4 & 3.4 & 3.8 \\
\hline PVH1 & 19.8 & 1.8 & 2.1 & 1.9 & & 368.0 & 6.2 & 8.6 & 12.8 & 5.4 & 8.4 & 2.9 & 6.9 & 7.8 \\
\hline PVH1 & 24.4 & 2.2 & 2.1 & 1.8 & & 637.3 & 10.5 & 11.7 & 20.0 & 8.6 & 11.7 & 4.5 & 10.7 & 12.0 \\
\hline PVH2 & 6.6 & 0.8 & 1.7 & 1.3 & & 74.0 & 1.2 & 1.5 & 2.8 & 1.6 & 5.0 & 0.7 & 1.7 & 1.9 \\
\hline $\mathrm{PVH} 2$ & 13.6 & 1.4 & 1.7 & 1.7 & & 200.3 & 3.8 & 4.2 & 7.3 & 3.4 & 6.6 & 2.0 & 4.8 & 5.4 \\
\hline PVH2 & 20.5 & 2.0 & 1.7 & 1.7 & & 473.9 & 8.1 & 10.3 & 15.5 & 7.0 & 10.4 & 3.8 & 9.1 & 10.3 \\
\hline $\mathrm{PVH} 2$ & 24.0 & 2.4 & 1.7 & 1.7 & & 823.2 & 13.9 & 16.3 & 22.9 & 10.8 & 15.5 & 5.9 & 14.3 & 16.1 \\
\hline
\end{tabular}

TAV, tricuspid aortic valve; BAV, bicuspid aortic valve; Circ, circular aortic valve; PHV, prosthetic heart valve; GOA, geometric orifice area; EOA, effective orifice area; $\mathrm{A}_{\mathrm{A}}$, unconstructed diameter; TKE, turbulent kinetic energy; SB, simplified Bernoulli; EB, extended Bernoulli; TP, turbulence production; SS, shear-scaling. 
Table 3. Summary of linear regression and Bland-Altman analysis

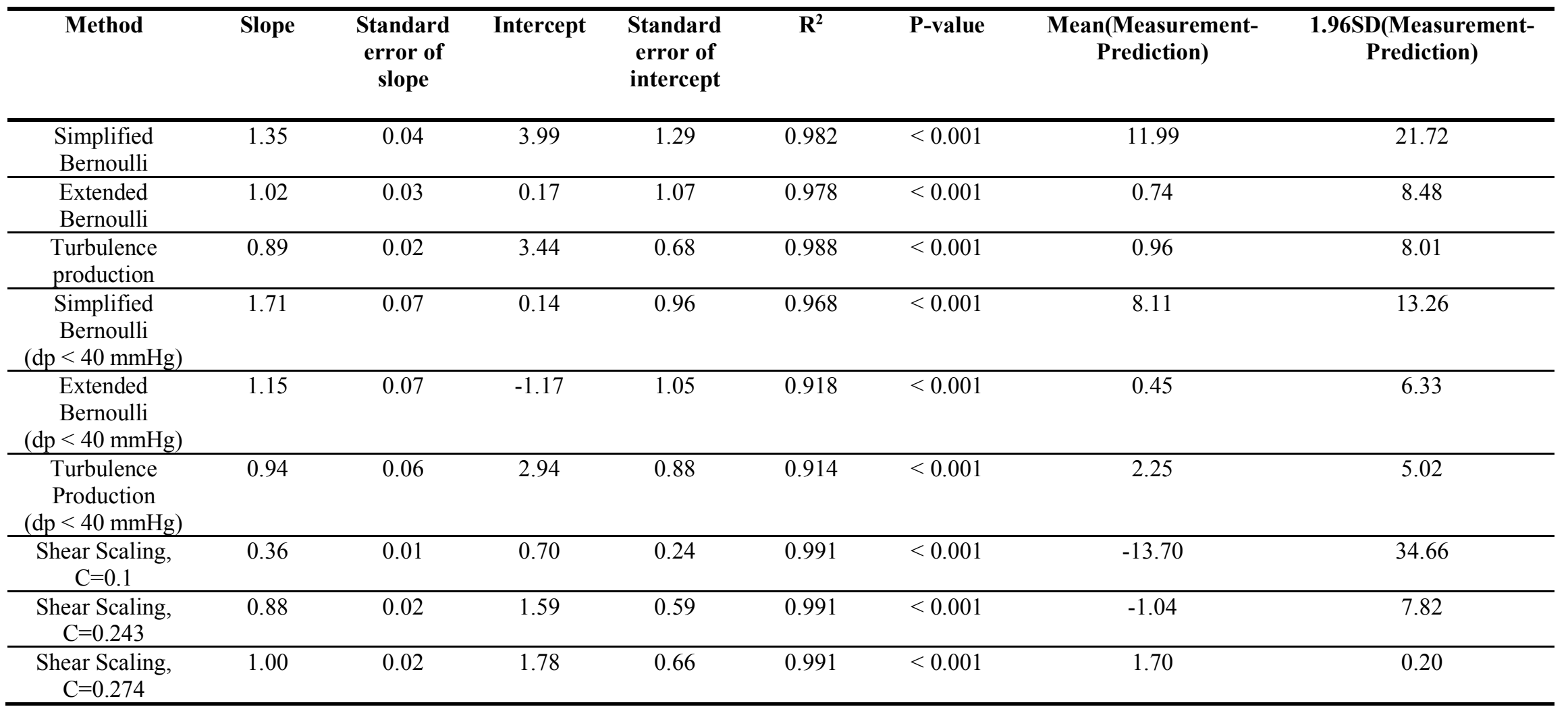


Supporting information captions

Supporting information 1. Supplementary figures and information 


\section{Validation of pressure drop assessment using 4D Flow MRI-based turbulence production in various shapes of aortic stenoses}

Hojin $\mathrm{Ha}^{1,2,3}$, John-Peder Escobar Kvitting ${ }^{2,3,4}$, Petter Dyverfeldt ${ }^{2,3}$, Tino Ebbers ${ }^{2,3}$

${ }^{1}$ Department of Mechanical and Biomedical Engineering, Kangwon National University, Chuncheon, Republic of Korea.

2Division of Cardiovascular Medicine, Department of Medical and Health Sciences, Linköping University, Linköping, Sweden.

${ }^{3}$ Center for Medical Image Science and Visualization (CMIV), Linköping University, Linköping, Sweden.

${ }^{4}$ Department of Cardiothoracic Surgery, Oslo University Hospital, Rikshospitalet, Oslo, Norway.

Running title: 4D Flow MRI-based turbulence production in stenoses.

Number of words: 4954 words

Number of items: 6 Figures, 3 Tables 
Velocity and turbulence production

The sectional velocity and turbulence production are shown in Figure S3a-c. The sectional visualization shows that each valve produces a unique velocity profile and turbulence production. Peak velocity and total turbulence production in this study ranged from $0.7 \mathrm{~m} / \mathrm{s}$ to $5.8 \mathrm{~m} / \mathrm{s}$ and from $74.0 \mathrm{~mW}$ to $5531.6 \mathrm{~mW}$, respectively (Figure S3). Both peak velocity and total turbulence production were closely correlated to the ratio of the flow rate to GOA (Q/GOA), while the geometric shape of the valve (e.g., triangular or circular shapes) did not show any influence on the correlation. The linear and quadratic regression curves were found as follows: Peak velocity $=1.08 \times(\mathrm{Q} / \mathrm{GOA})+0.19$ and Turbulence Production $=$ $0.22 \times(\mathrm{Q} / \mathrm{GOA})^{2}-0.26 \times(\mathrm{Q} / \mathrm{GOA})+0.22$ (Figure S3). 

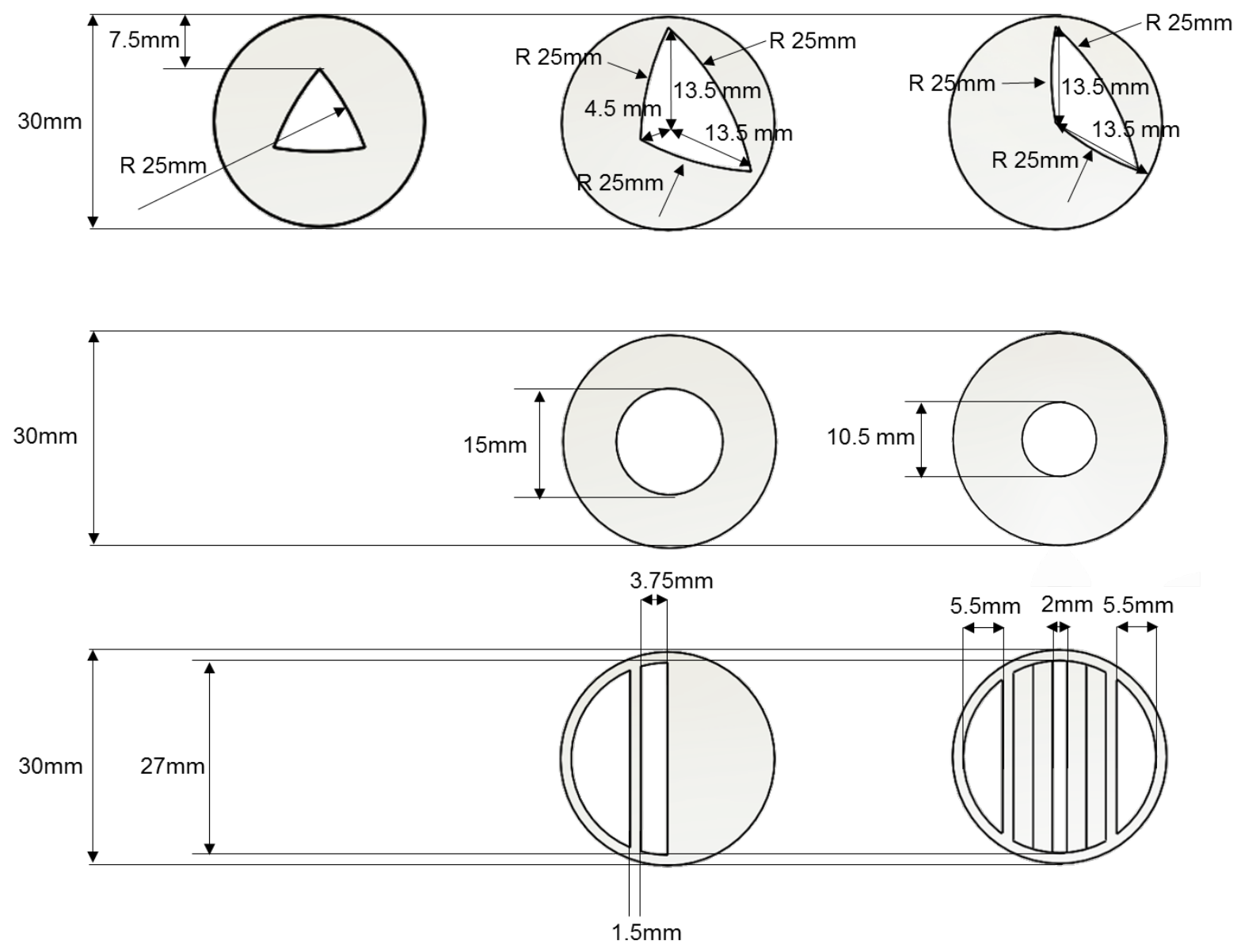

Figure S1. Detailed geometric dimensions of the aortic heart valves used in this study. 
a

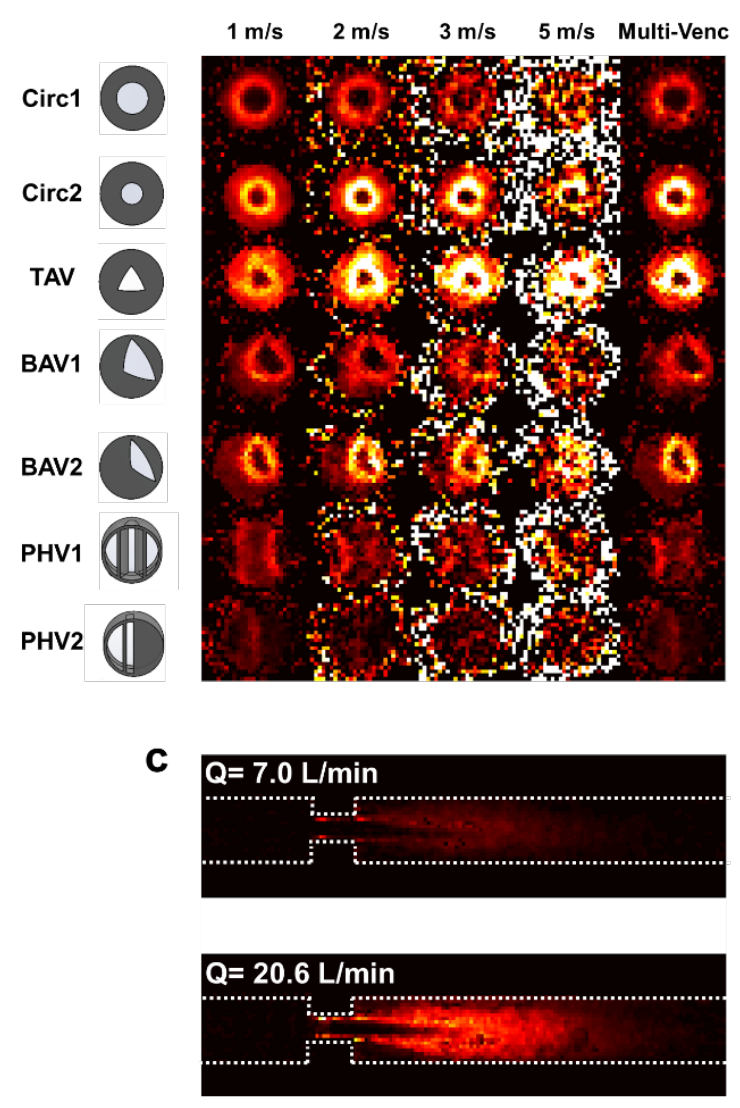

b

$1 \mathrm{~m} / \mathrm{s} \quad 2 \mathrm{~m} / \mathrm{s} \quad 3 \mathrm{~m} / \mathrm{s} \quad 5 \mathrm{~m} / \mathrm{s}$ Multi-Venc
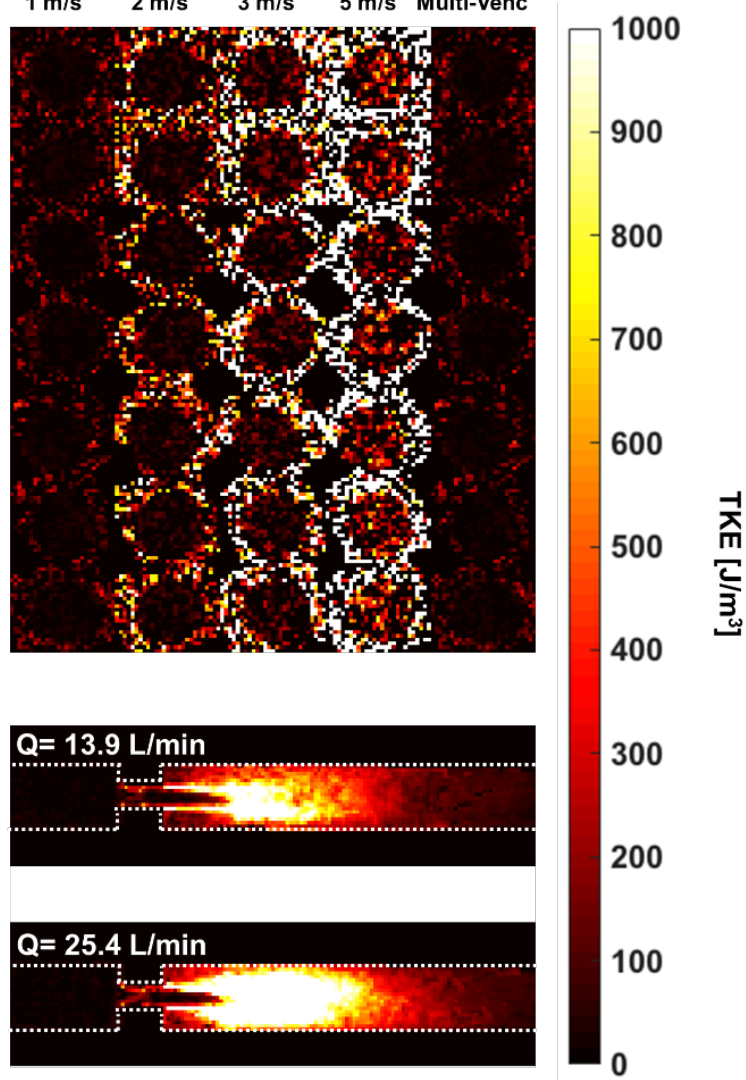

Figure S2. Turbulent kinetic energy (TKE) at various VENC and multi-VENC measurements. (a) TKE at post-valve region (X/D = 1), (b) TKE at inlet flow region (X/D = -1) and (c) TKE distribution at various flow rates. $\mathrm{X}$ and $\mathrm{D}$ indicate an axial distance from the heart valve and a diameter of the channel, respectively. A flow rate of $\sim 25.2 \mathrm{~L} /$ min was used in (a) and (b). TAV was used at (c). 
a

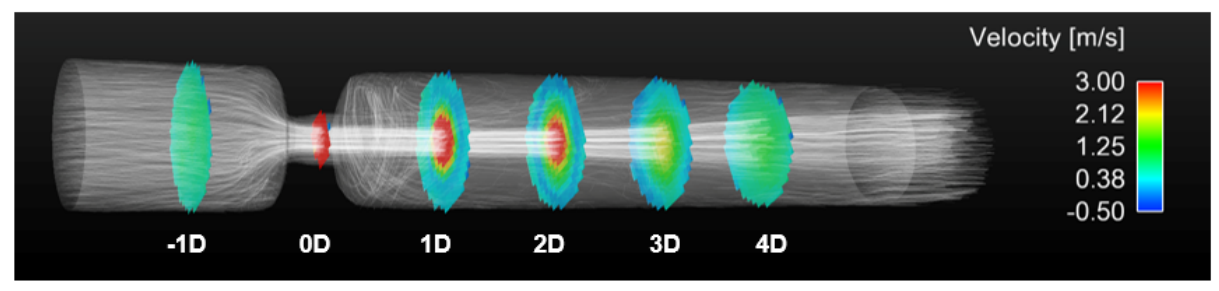

b

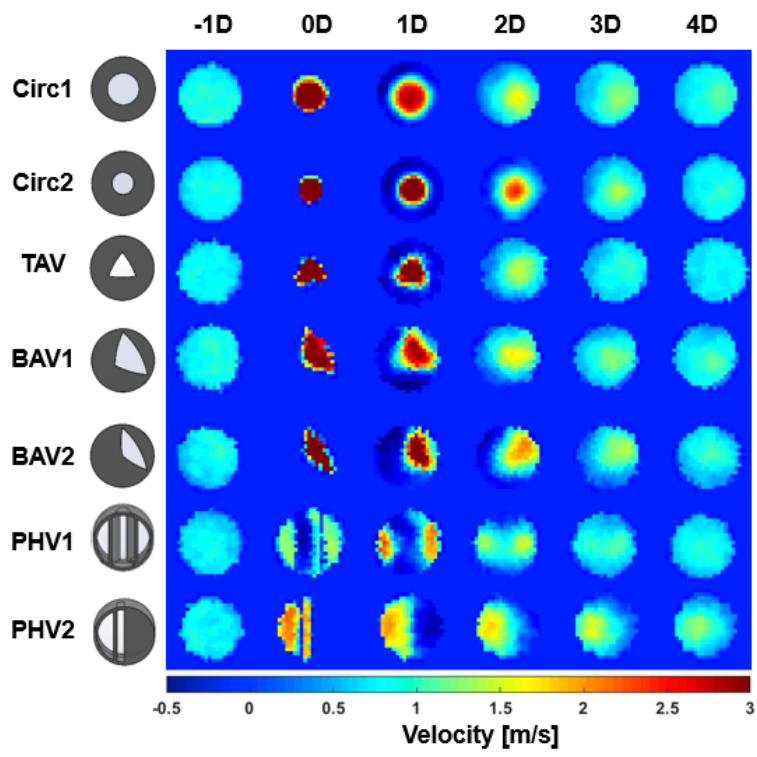

C

$\begin{array}{llllll}-1 D & 0 D & 1 D & 2 D & 3 D & 4 D\end{array}$

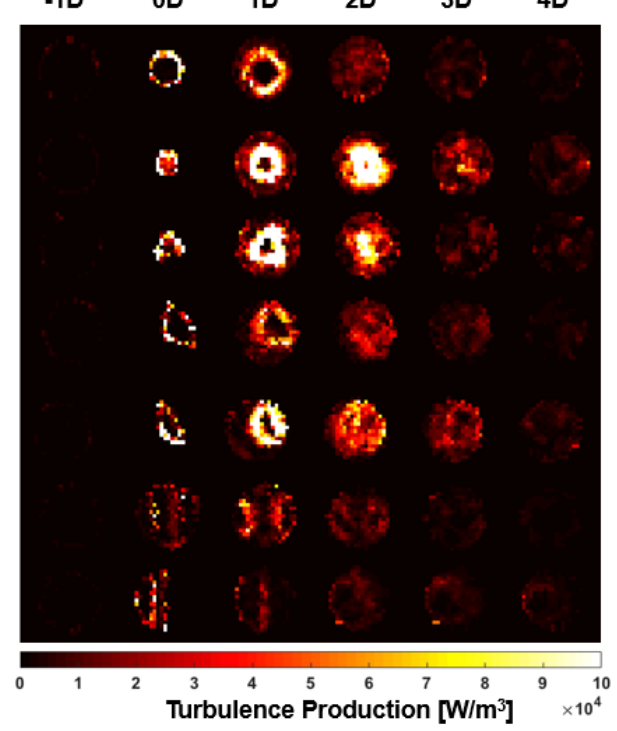

Figure S3. 4D Flow MRI visualization of velocity and turbulence production at various stenotic heart valves. (a) Representative visualization of streamline and sectional velocity distribution with Circ2 valve, (b) sectional velocity distribution, (c) sectional turbulence production. A flow rate of $\sim 25.2 \mathrm{~L} / \mathrm{min}$ was used in (a-c). 
a

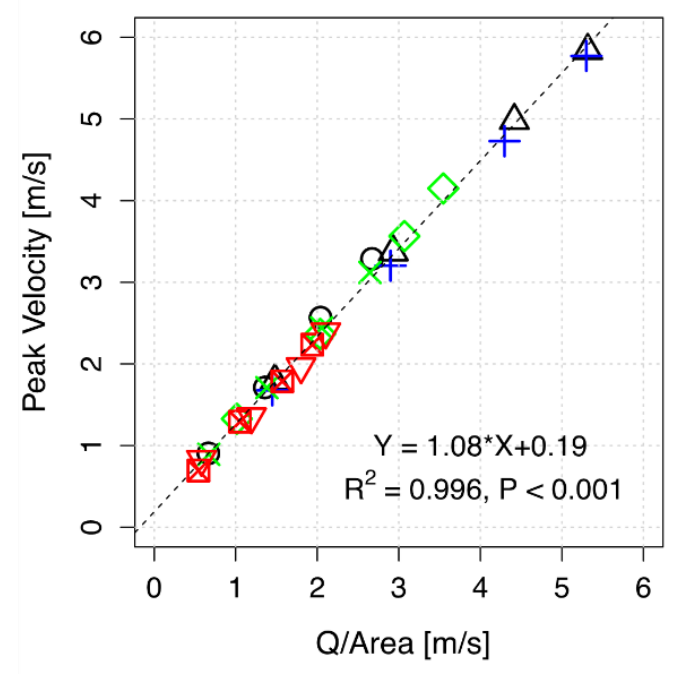

b

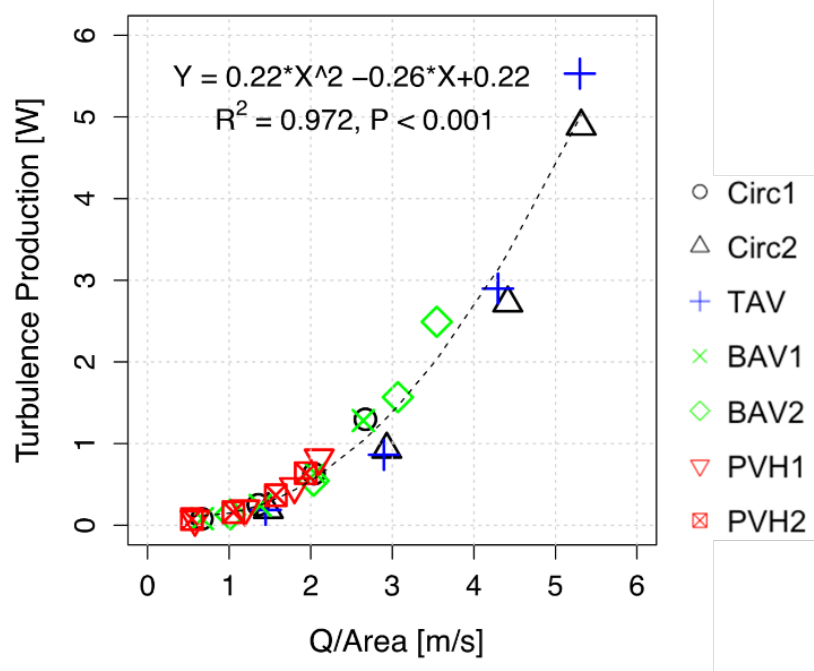

Figure S4. 4D Flow MRI visualization of velocity and turbulence production at various stenotic heart valves. (a) Relationship between peak velocity and expected peak velocity (Q/area) and (b) relationship between turbulence production and expected peak velocity (Q/area). 
a

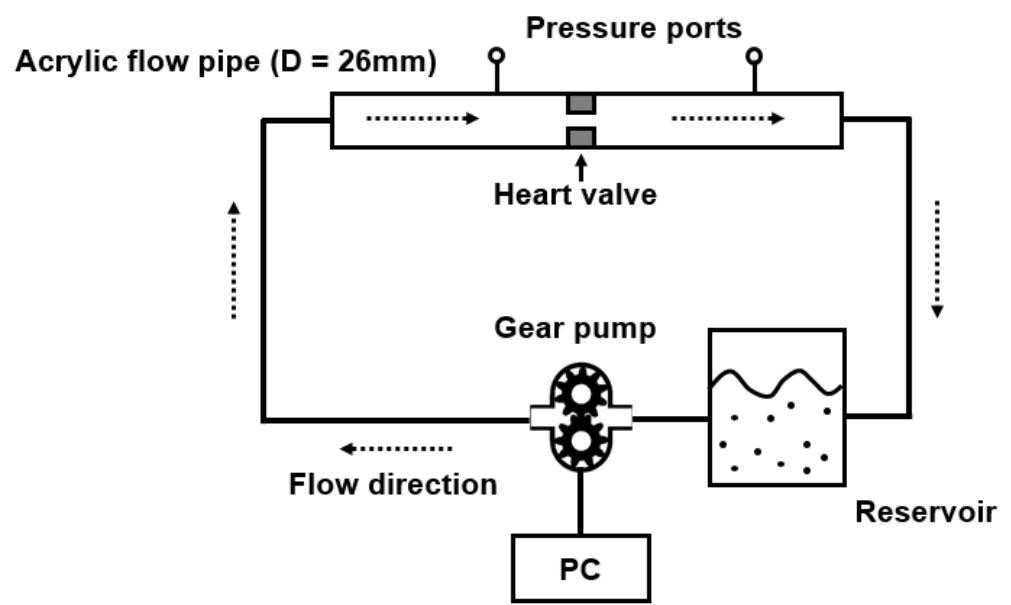

3D Printed heart valves
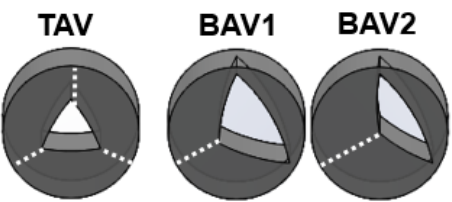

Circ1

Circ2

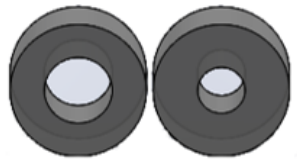

PHV1

PHV2

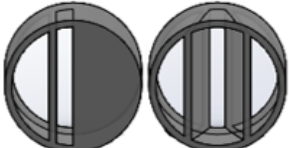

Figure 1

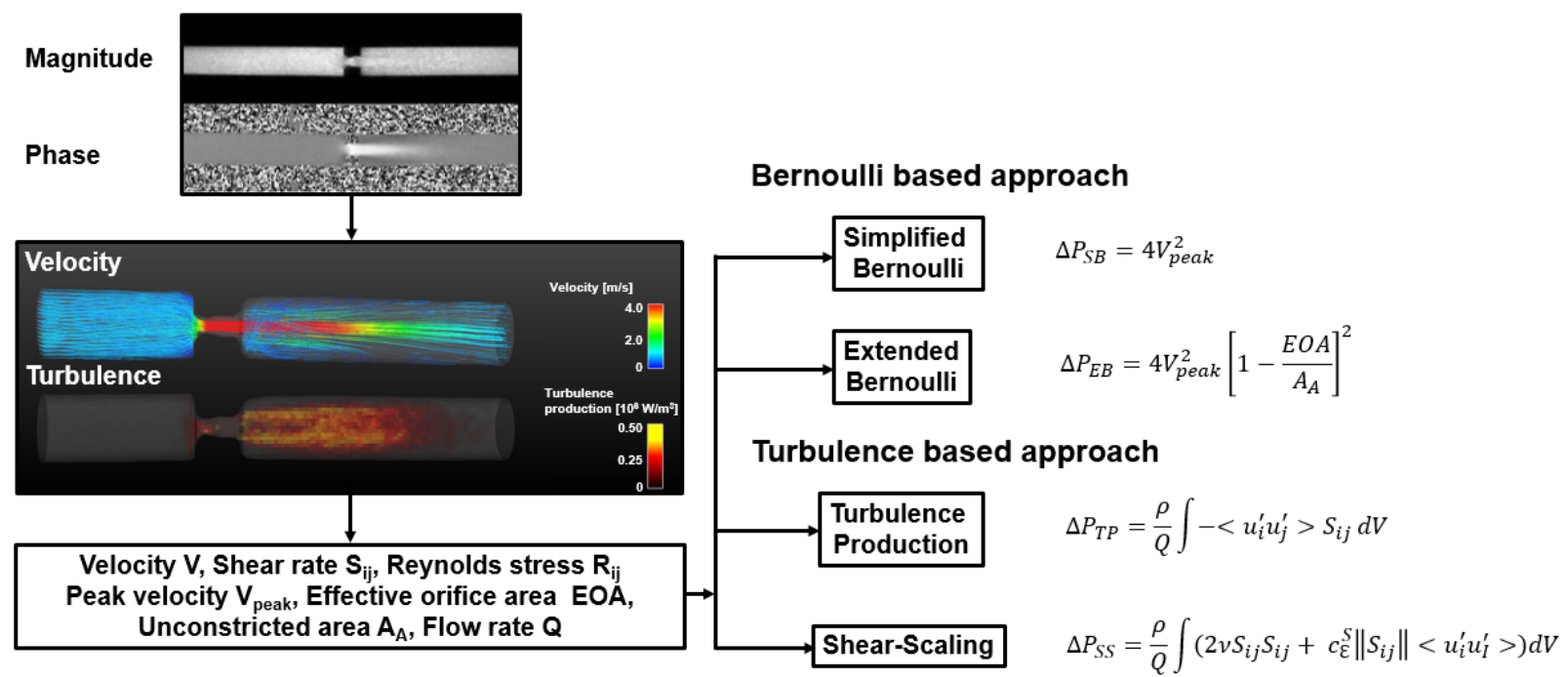

Figure 2 


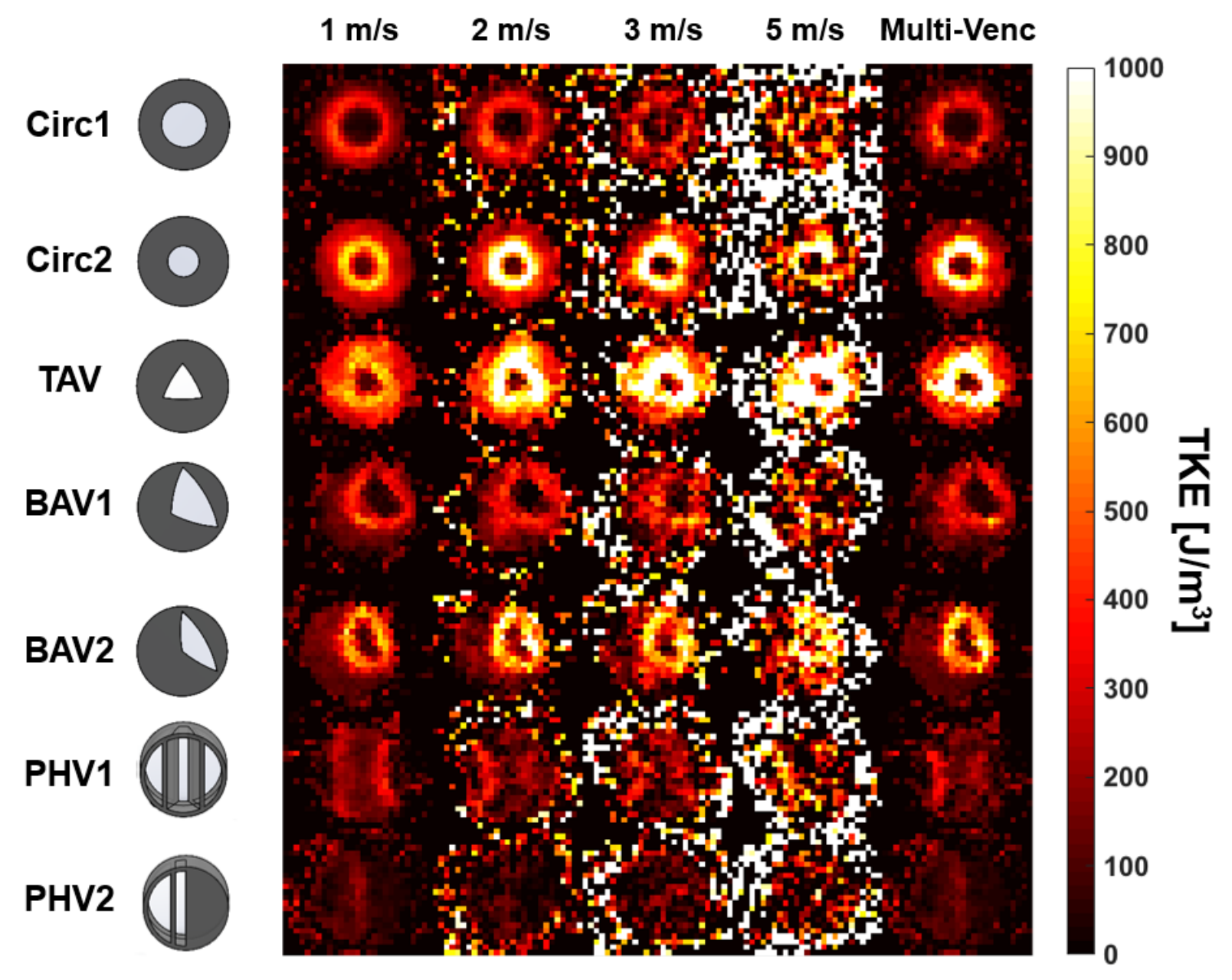

Figure 3

a

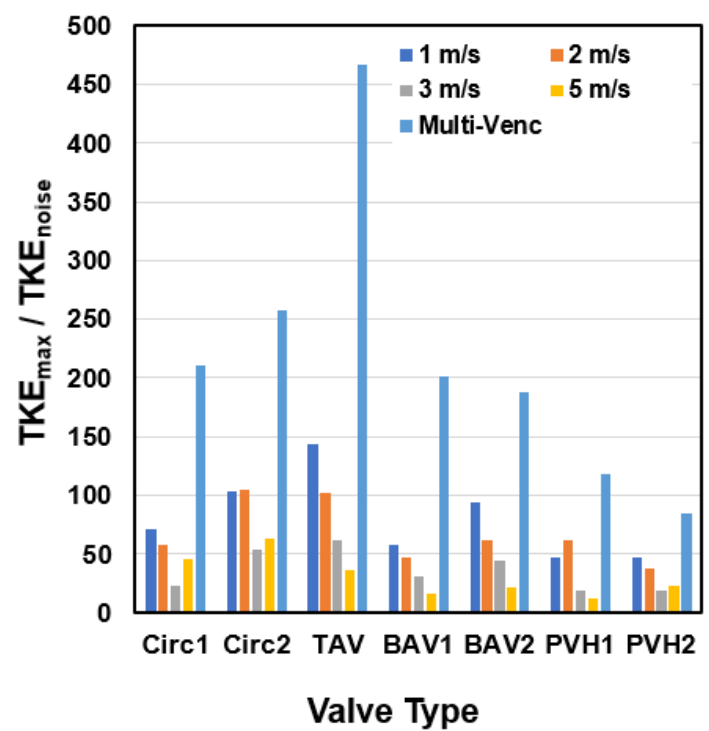

Figure 4 b

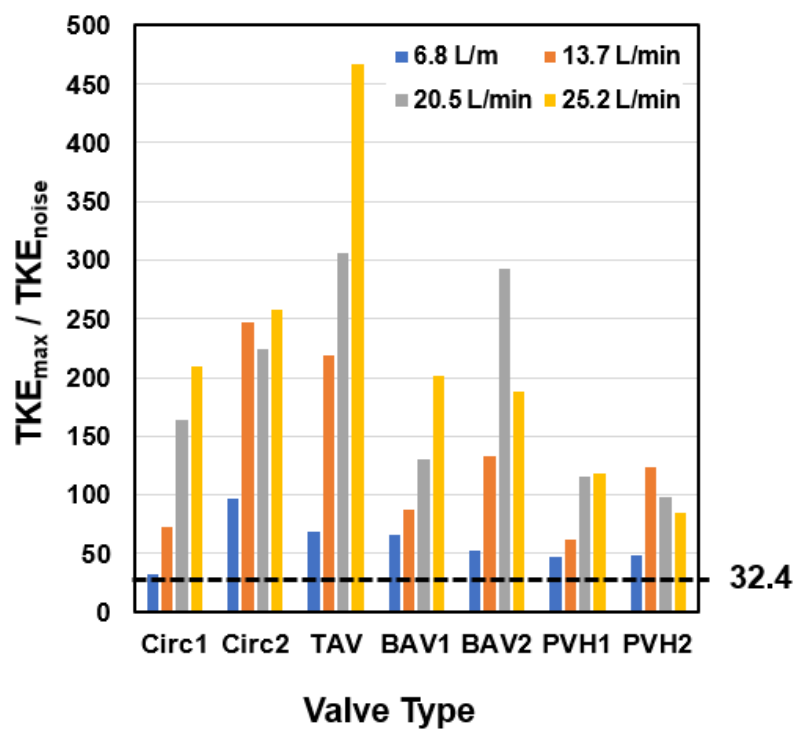


SB

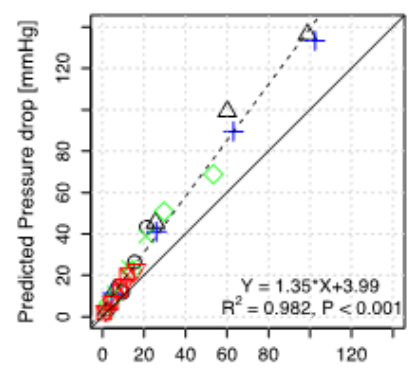

a

Measured Pressure drop [mmHg]

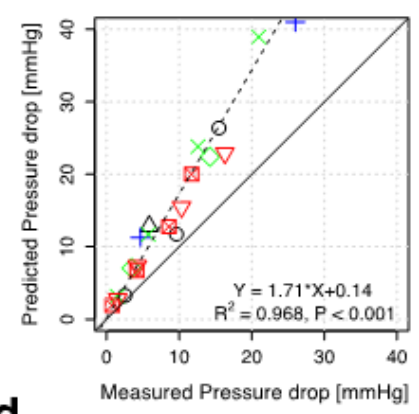

d

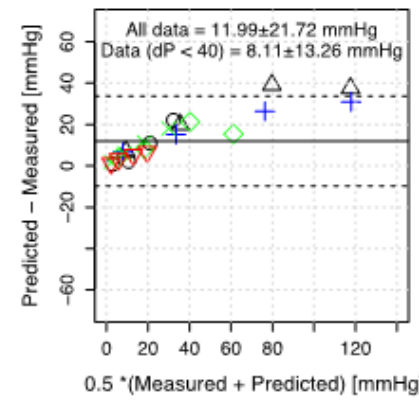

g

Figure 5
EB

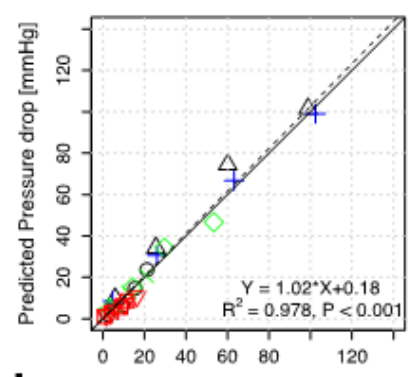

b Measured Pressure drop [mmHg]
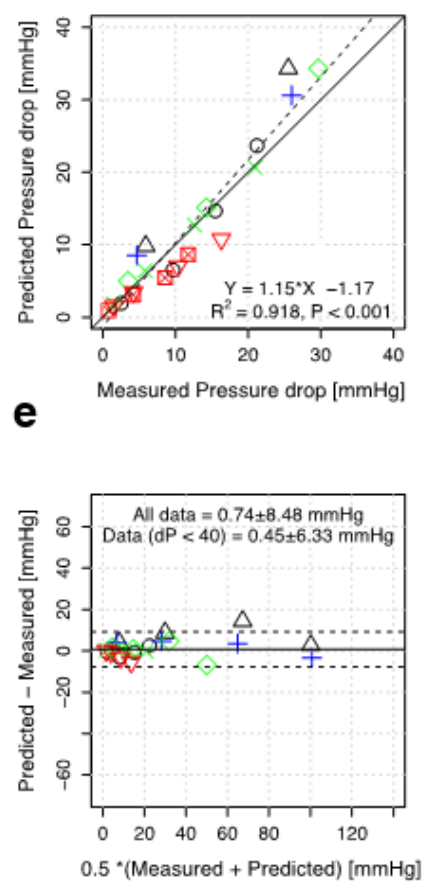

h
TP

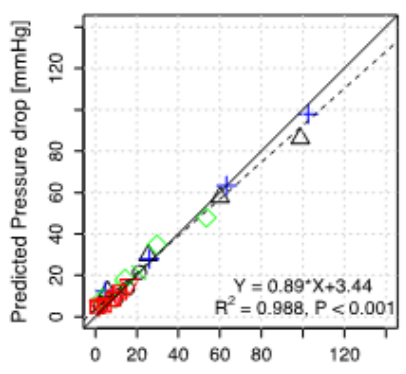

C Measured Pressure drop [mmHg]
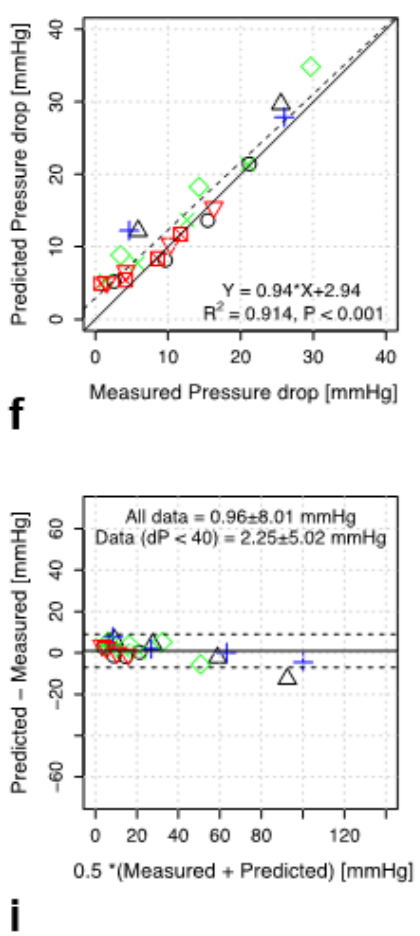

- Circ1

$\triangle$ Circ2

+ TAV

BAV1

BAV2

$\checkmark \mathrm{PVH} 1$

$\bowtie$ PVH2 

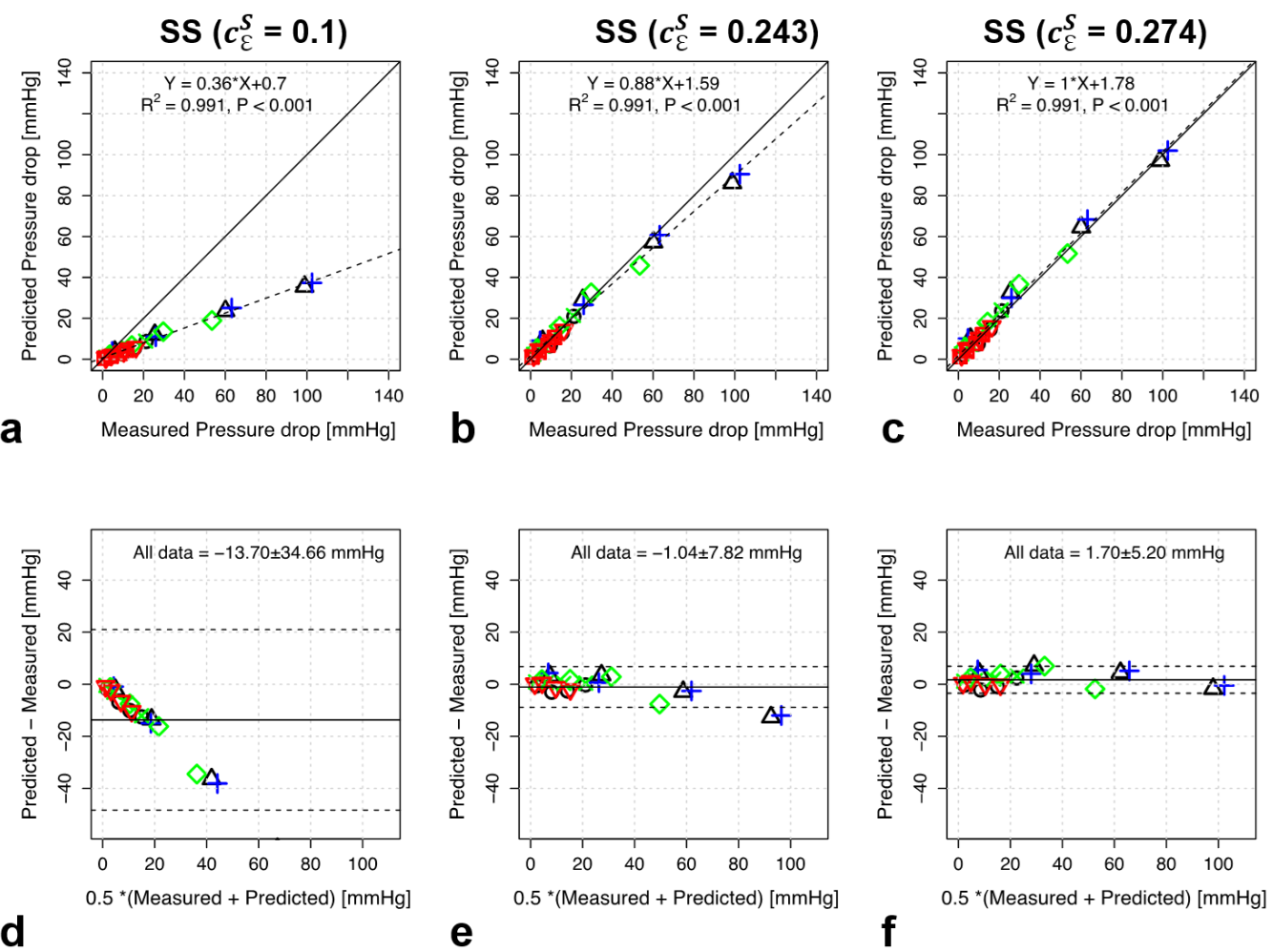
- Circ1
$\triangle$ Circ2
+ TAV BAV1 BAV2
$\checkmark \mathrm{PVH} 1$
$\otimes \mathrm{PVH} 2$

d

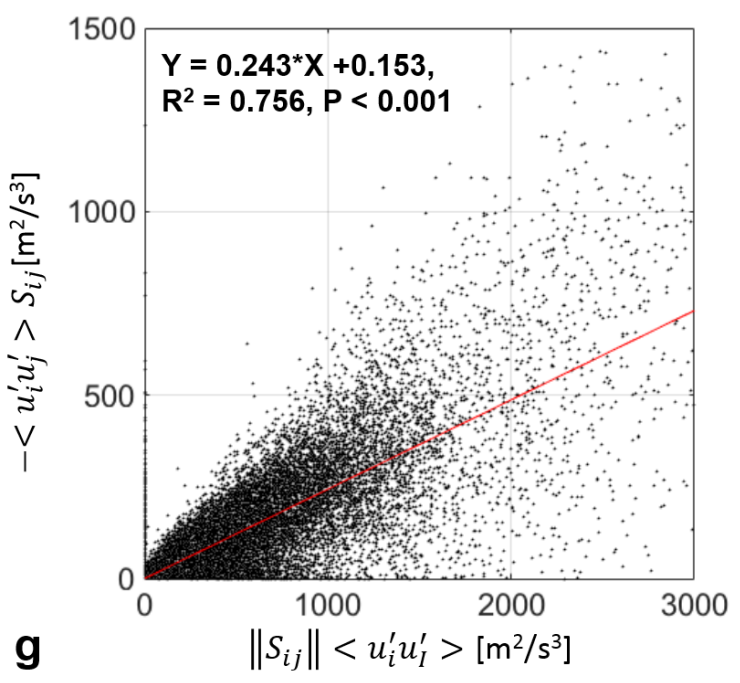

Figure 6 